University of Nebraska - Lincoln

DigitalCommons@University of Nebraska - Lincoln

Timothy J. Gay Publications

Research Papers in Physics and Astronomy

October 2001

\title{
Monte Carlo studies of Mott scattering asymmetries from gold foils
}

\author{
M. A. Khakoo \\ California State University, Fullerton, California \\ D. Roundy \\ University of California, Berkeley, California \\ C. Hicks \\ Esperanza High School, Yorba Linda, California
}

N. Margolis

Tustin High School, Tustin, California

E. Yeung

Glenn Wilson High School, Hacienda Heights, California

See next page for additional authors

Follow this and additional works at: https://digitalcommons.unl.edu/physicsgay

Part of the Physics Commons

Khakoo, M. A.; Roundy, D.; Hicks, C.; Margolis, N.; Yeung, E.; Ross, A. W.; and Gay, Timothy J. , "Monte Carlo studies of Mott scattering asymmetries from gold foils" (2001). Timothy J. Gay Publications. 48.

https://digitalcommons.unl.edu/physicsgay/48

This Article is brought to you for free and open access by the Research Papers in Physics and Astronomy at DigitalCommons@University of Nebraska - Lincoln. It has been accepted for inclusion in Timothy J. Gay Publications by an authorized administrator of DigitalCommons@University of Nebraska - Lincoln. 


\section{Authors}

M. A. Khakoo, D. Roundy, C. Hicks, N. Margolis, E. Yeung, A. W. Ross, and Timothy J. Gay 


\title{
Monte Carlo studies of Mott scattering asymmetries from gold foils
}

\author{
M. A. Khakoo \\ Physics Department, California State University, Fullerton, California 92834 \\ D. Roundy \\ Chemistry Department, University of California, Berkeley, California 92222 \\ C. Hicks* \\ Esperanza High School, Yorba Linda, California 92670 \\ N. Margolis ${ }^{\dagger}$ \\ Tustin High School, Tustin, California 92780 \\ E. Yeung* \\ Glenn Wilson High School, Hacienda Heights, California 91745 \\ A. W. Ross \\ Department of Physics, University of Texas, Austin, Texas 78712 \\ T. J. Gay \\ Behlen Laboratory of Physics, University of Nebraska, Lincoln, Nebraska 68588 \\ (Received 9 March 2001; published 12 October 2001)
}

\begin{abstract}
We present the results of a series of model Monte Carlo calculations of the scattering of spin-polarized electrons from gold foil targets. Our calculations examine the behavior of the left-right scattering asymmetry A as a function of various parameters conventionally used in extrapolation of the left-right asymmetry to singleatom and/or elastic scattering conditions. These parameters include target thickness, scattered count rate, and the maximum energy that an electron can have lost in the target and still be detected. Data are obtained at incident electron energies of $10-120 \mathrm{keV}$, with detector-subtended half-cone angles of $5^{\circ}, 10^{\circ}$, and $20^{\circ}$, and gold foils of average thickness varying from 3 to $1000 \AA$. Both elastic and inelastic electron scattering effects have been considered. Comparisons of our results are made with existing measurements and theoretical models. We make recommendations concerning extrapolation algorithms and for future experiments to test the present Mott scattering Monte Carlo model.
\end{abstract}

DOI: 10.1103/PhysRevA.64.052713

PACS number(s): $34.80 . \mathrm{Nz}, 39.10 .+\mathrm{j}$

\section{INTRODUCTION}

The standard method of measuring the spin polarization of a collimated electron beam involves Mott scattering from high- $Z$ targets $[1]$. Gold $(Z=79)$ is the most popular target material, as it is easy to obtain and to make into foils. It is also inert and thus nonoxidizing. Thorium and uranium, having a higher $Z$, provide a higher sensitivity, but they are not as accessible as gold and are not as easily fabricated into foils. While recent improvements in measuring the analyzing power of a Mott polarimeter have been made by Gellrich and Kessler, who used double-scattering techniques [2], efforts still need to be made to make Mott polarimetric measurements absolute in the more common and easier-to-use singlescattering setups. In the last 20 years, experimental studies to improve the accuracy of single-scattering Mott polarimeters

\footnotetext{
*Present address: Physics Department, California Institute of Technology, Pasadena, CA 91125.

${ }^{\dagger}$ Present address: Physics Department, Stanford University, Stanford, CA 94305.
}

have been made by Mayer et al. [3], Gay et al. [4], Fletcher, Gay, and Lubell [5], Jost [6], Hodge et al. [7], and Campbell et al. [8]. These investigators used both high-energy conventional and concentric-cylinder Mott polarimeters. Similarly, more compact (spherical-type) "mini-Mott" polarimeters have been developed by Dunning and co-workers $[9,10]$.

This paper addresses experimental problems associated with the accurate extrapolation of single Mott scattering asymmetries $A$ to their "true" value ( $\equiv A_{\text {true }}$ ), which is associated with elastic scattering from a single atom. Our work is motivated by the empirical studies of Gay et al. [4], Fletcher Gay, and Lubell [5], and Campbell et al. [8]. These authors investigated the behavior of $A$ as a function of foil thickness $(t)$, the maximum energy an electron can have lost in the foil and still be detected $(\varepsilon)$, and the scattered electron count rate $(N)$. They also considered the theoretical bases used in extrapolating measured values of $A$ to $A_{\text {true }}$. In the most recent analyses by Gay et al. [4] the following conclusions regarding extrapolation methods were reached.

(i) Deviation of $A$ from $A_{\text {true }}$ is "due to plural elastic scattering compounded with small-angle inelastic multiple scattering." "Plural" scattering involves several large-angle 
scattering events, whereas "multiple"scattering involves a large number of small-angle collisions.

(ii) The appropriate procedure for obtaining $A_{\text {true }}$ is an $A$ vs $t$ extrapolation at any $\varepsilon$ value with a foil thickness range such that $A$ vs $t$ is demonstrably linear, i.e., the extrapolated value of $A$ at $t=0$ is independent of any fitting function that is first order in $t$. This follows from their observation that elastic plural scattering is the dominant process that reduces $A$. forms

(iii) In the event that $A$ vs $t$ is nonlinear, the functional

$$
A(t)=a_{1}+b_{1} e^{-\Gamma t}
$$

and

$$
A(t)=a_{2}+b_{2} N(t)
$$

were recommended. Here $a_{1}, b_{1}, a_{2}, b_{2}$, and $\Gamma$ are constants for a given $\epsilon$ and incident electron energy $E$. This follows from the observation that, even at values of $E$ where $A$ vs $t$ plots showed nonlinear behavior, $A$ vs $N$ plots were essentially linear, and yielded the best reduced chi-squared $\left(\chi_{\nu}^{2}\right)$ fit.

(iv) Under the conditions where $A$ vs $t$ plots showed large nonlinear behavior (e.g., at low $E$ ), $N$ values were significantly removed from the $N=0$ axis. This factor reduced the precision of $A$ vs $N$ extrapolations in determining $A_{\text {true }}$.

(v) In Eq. (1) $\Gamma$ is approximately equal to the inelastic mean free path $\left(\lambda_{i}\right)$ of electrons in the foil.

By making a Monte Carlo study of Mott scattering, where $A_{\text {true }}$ is a known quantity, we can obtain insights into the empirical extrapolation forms used to date. Moreover, the physical assumptions used in the model calculations can be altered, and the resulting changes in the dependence of $A$ on $N, \varepsilon$, and $t$ analyzed, providing further insights into the foil scattering physics. The goal of this work is to provide such insights, and to critically evaluate the conclusions reached in earlier investigations.

\section{GENERAL EXPERIMENTAL CONSIDERATIONS}

In the conventional Mott electron polarimetry experiments addressed here, a collimated beam of transversely spin-polarized electrons is scattered by atoms in a foil or bulk target. The experiment measures the left-right electron scattering intensity asymmetry $A$ for polar electron scattering angles centered about $\theta$ (typically $120^{\circ}$ ), at azimuthal angles centered about $\phi=0^{\circ}$ and $180^{\circ}$, as shown schematically in Fig. 1. With electrostatic retarding grids placed in front of the detectors, one can also control $\varepsilon$, the maximum energy loss electrons can have suffered in the target and still reach the detector. The observed left-right asymmetry of scattered electrons, a measure of the difference in electron fluence scattered to the left $\left(N_{L}\right)$ and right $\left(N_{R}\right)$, is directly related to the transverse electron spin polarization normal to the scattering plane, $P_{n}$, by [11]

$$
A \equiv \frac{N_{L}-N_{R}}{N_{L}+N_{R}}=P_{n} S_{\mathrm{eff}}(E, t, \theta, \Delta \Omega, \varepsilon),
$$

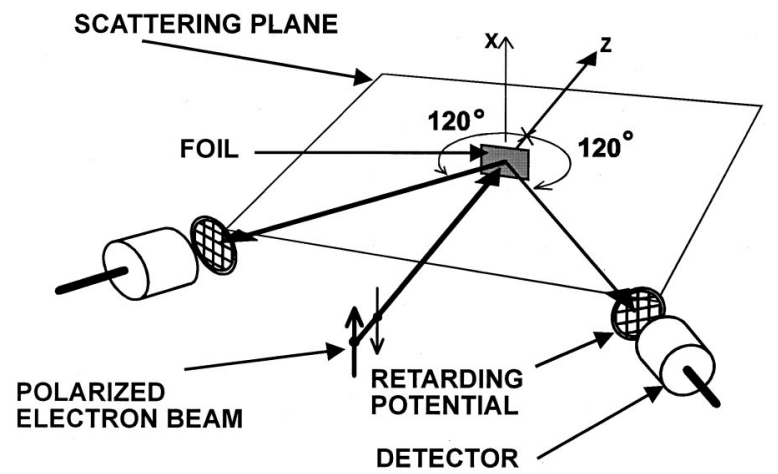

FIG. 1. Schematic drawing of a conventional Mott scattering apparatus using retarding potential discrimination of scattered electrons.

where $S_{\text {eff }}$ is the "effective" Sherman function for the complete polarimeter (including the electron detection system). The value of $A$ depends on $E$, the foil thickness $t$, the solid angle of the detectors $\Delta \Omega$, and $\varepsilon$. In the limit of $t \rightarrow 0$ and $\varepsilon \rightarrow 0, S_{\text {eff }} \longrightarrow S(\theta, \Delta \Omega)$, the elastic single-scattering Sherman function averaged over the detector solid angle. Since plural and multiple scattering degrade the observed left-right asymmetry, $S_{\text {eff }}<S$. The effects of plural/multiple scattering in solid targets are complicated to model analytically, but the Monte Carlo method is ideally suited to investigate such processes. In the present analysis, we ignore effects due to instrumental asymmetries [1].

\section{THEORETICAL BACKGROUND}

\section{A. General}

The quantum-mechanical description of the scattering of spin-polarized electrons by single atoms requires the solution of the relativistic Dirac equation [11]. The scattering is described by direct and spin-flip differential scattering amplitudes $f(\theta)$ and $g(\theta)$, respectively. Our model uses $I, S, T$, and $U$ parameters, which are derived from these scattering amplitudes and their complex conjugates:

$$
\begin{gathered}
I(\theta)=|f|^{2}+|g|^{2}, \quad S(\theta)=\frac{i\left(f g^{*}-f^{*} g\right)}{|f|^{2}+|g|^{2}}, \\
T(\theta)=\frac{|f|^{2}-|g|^{2}}{|f|^{2}+|g|^{2}}, \quad U(\theta)=\frac{f g^{*}+f^{*} g}{|f|^{2}+|g|^{2}} .
\end{gathered}
$$

Thus $I(\theta) \equiv d \sigma(\theta) / d \Omega$ is the elastic spin-averaged differential scattering cross section (DCS). The Sherman function $S(\theta)$ is defined above. The DCS with polarized electrons can be related to $I(\theta)$ :

$$
\frac{d \sigma(\theta, \phi)}{d \Omega}=I(\theta, \phi)=I(\theta)\left(1-S(\theta) P_{n} \sin \phi\right) .
$$

Figure 2 illustrates the electron-atom scattering geometry. The unit vector $\hat{\mathbf{n}}$ is perpendicular to the scattering plane defined by $\mathbf{k}$ and $\mathbf{k}^{\prime}$, the incident and scattered electron momenta, i.e., $\hat{\mathbf{n}}=\hat{\mathbf{k}} \times \hat{\mathbf{k}}^{\prime}$. The polarization vector of the incident 


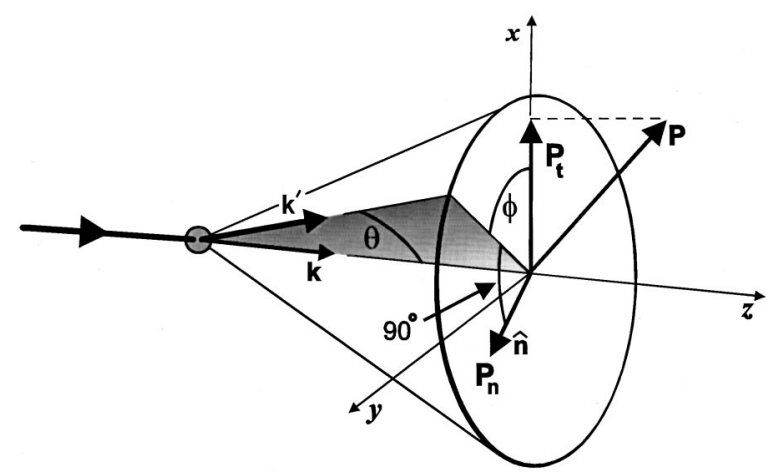

FIG. 2. Electron spin and momenta vector diagrams relevant to Mott scattering (see text). $\quad P_{n}$ is the component of $\mathbf{P}$ perpendicular to the scattering plane of the electron, defined by $\mathbf{k}$ and $\mathbf{k}^{\prime}$. The transverse polarization $P_{t}$ is the component of the electron spin polarization vector $\mathbf{P}$ perpendicular to the experimentally configured $y z$ scattering plane in Fig. 1. $\mathbf{P}$ is initially set parallel to $\hat{\mathbf{x}}$ in our model.

electron, $\mathbf{P}$, has a component $P_{n}$ along $\hat{\mathbf{n}}$. The scattered electron polarization $\mathbf{P}^{\prime}$ is given by [11]

$$
\mathbf{P}^{\prime}=\frac{\left.\left[P_{n}+S(\theta)\right] \hat{\mathbf{n}}+T(\theta) \hat{\mathbf{n}} \times(\mathbf{P} \times \hat{\mathbf{n}})+U(\theta)(\hat{\mathbf{n}} \times \mathbf{P})\right]}{1+\mathbf{P} \cdot \hat{\mathbf{n}}(\theta)} .
$$

We use this equation to transform the components of $\mathbf{P}$ into $\mathbf{P}^{\prime}$ in terms of the body-centered coordinate system $\left\{\hat{\mathbf{n}}, \hat{\mathbf{k}}^{\prime}, \hat{\mathbf{n}} \times \hat{\mathbf{k}}^{\prime}\right\}$ used after scattering from the initial (framecentered) coordinates $\hat{\mathbf{n}}, \hat{\mathbf{k}}$, and $\hat{\mathbf{n}} \times \hat{\mathbf{k}}$.

\section{B. Previous theoretical models}

Mott scattering in extended targets has been studied analytically by only a few authors, who were forced by the complexity of the problem to consider only elastic scattering $[12-15]$. With the advent of high-speed computers, the Monte Carlo method of tracking electron trajectories is the best way to model scattering in foils, but to date it has only been used for polarized electron scattering in two reports to our knowledge, those of Hnizdo [16] and Qiao and Kakizaki [17]. Hnizdo's calculations consider only elastic scattering, and use only the target thickness as a parameter on which $A$ can depend. Qiao and Kakizaki consider inelastic scattering in an $a d$ hoc fashion and as such can study $A$ vs $\varepsilon$ as well as $t$. In the case of analytic studies, the behavior of $A$ as a function of $t$ has been considered by Wegener [12]. Extensions of this work were made by Wegener [13], Greenberg et al. [14], and Braicovich and de Michelis [15]. They used $S, T, U$, and $I$ from the screened-Coulomb calculations of Holzwarth and Meister [18]. Owing to the complexity in handling higher-order processes, these analytic investigations were limited to double scattering only. Singularities in integrals occurring from contributions of electrons scattered into $\theta=90^{\circ}$, i.e., the plane of the foil, were suppressed by distributing these electrons over a finite range about the foil plane as determined by multiple-scattering considerations. The Wegener analysis yielded a functional dependence of $A$ on $t$ with the form

$$
A(t) \approx \frac{A_{\text {true }}}{1+b_{3}(E, \theta) t},
$$

where $b_{3}$ is a constant.

The analytic method has the following disadvantages: (i) It is limited to large $E$ and small $t$ values, where double scattering is a small first-order correction. (ii) It does not explicitly consider inelastic scattering, which can be an important process. (iii) It cannot be easily modified to take into account the effect of finite-size detectors.

Further progress was made by Hnizdo [16] also using the $S, T, U$, and $I$ values from Ref. [18]. Hnizdo developed a Monte Carlo algorithm in the energy range from $E$ $=46-290 \mathrm{keV}$, with random-walk statistics (Gaussian spreading) to model multiple elastic electron scattering. For multiple scattering, the electron is first deflected using a random-walk algorithm without change of polarization. The resulting Gaussian probability distribution $G(\theta)$ is characterized by a standard deviation spreading $\left\langle\theta^{2}\right\rangle$ :

$$
G(\theta)=\frac{2 \theta}{\left\langle\theta^{2}\right\rangle} \exp \left(\frac{-\theta^{2}}{\left\langle\theta^{2}\right\rangle}\right)
$$

where

$$
\left\langle\theta^{2}\right\rangle=n l \int_{0}^{\Theta} \theta^{2} I(\theta) 2 \pi \sin (\theta) d \theta
$$

and

$$
\int_{0}^{\pi} G(\theta) d \theta=1
$$

Here, $n$ is the number density of atoms and $l$ is the energydependent elastic scattering path length of the electron in the foil. The electrons could suffer two distinct types of elastic scattering: multiple scattering through an angle $\theta<\Theta$, and plural scattering through the angle $\theta>\Theta$. The critical angle $\Theta$, which demarcated these two scattering zones, was selected to be $20^{\circ}$ for all incident energies, because the model was found to be insensitive to $\Theta$ around this value.

In Hnizdo's Monte Carlo algorithm, the electron undergoes numerous large-angle scattering events separated by paths along which multiple scattering occurs. At each largeangle scattering site, the probability of the electron reaching the detector, a differential probability element $\Delta P$, is determined from

$$
\Delta P(\delta, \theta, \phi)=W \frac{1}{\sigma_{e}} \frac{d \sigma(\theta, \phi)}{d \Omega} \exp \left(-n \sigma_{e} \delta\right),
$$

where $\sigma_{e}$ is the total elastic scattering cross section and $\delta$ is the path length in the foil from the scattering point to the detector. The quantity $W$ is a statistical weighting factor that decreases exponentially with the total path length that the electron travels in the foil. The $\Delta P$ 's are summed for both 
detectors as the electron moves through the foil. A further correction in $\Delta P$ was made for multiple-scattering processes that could have occurred on the way out to the detector. Only for plural scattering $(\theta>\Theta)$ was the orientation of the electron's spin changed, using Eq. (6). For multiple scattering $(\theta<\Theta)$, the polarization was rotated using a linear approximation to Eq. (6) depending on the multiple-scattering angle $\theta[16]$. The electron was forced to remain in the foil until its running statistical weight $W$ was reduced to a minimum preset value. The final summed $\Delta P(\delta, \theta, \phi)$ values were then used to calculate $A$.

There are several problems with Hnizdo's method.

(i) It is clear that Hnizdo's propagation of detection probabilities is unphysical. Realistically, an electron has one chance of detection, and thus cannot be modeled by adding sequential $\Delta P(\delta, \theta, \phi)$ while it remains in the foil.

(ii) While $\Delta P$ for electrons plurally scattered toward the detector is reduced by a multiple-scattering correction, Hnizdo's calculation does not take into account the possibility of multiple scattering into the detector's solid angle of acceptance. [Note that Eq. (9) does not include $\Delta \Omega$.]

(iii) The direction of electron spin after a collision is corrected accurately only for plural scattering and not for multiple scattering where a linear transformation algorithm is used. However, a large number of multiple scatterings with small changes in electron spin polarization can accumulate geometrically to cause significant changes in $A$. This effect was also discussed by Qiao and Kakizaki [17].

(iv) The addition of multiple scattering (with a linear polarization change) during the electron's travel to a pluralscattering event with exact polarization change may pose problems in thin foils, since a single plural scattering may occur more often depending on the inelastic scattering mean free path $\lambda_{i}\left(=1 / n \sigma_{i} ; \sigma_{i}\right.$ is the total inelastic cross section).

(v) Finally, scattering of the electron via inelastic processes in the foil was not investigated.

The more recent calculations of Qiao and Kakizaki [17] are similar to those of Hnizdo, but they make improvements on Hnizdo's calculations by including the effect of inelastic scattering in a phenomenological way using a mean energy loss range in their Monte Carlo algorithm. They also improve on Hnizdo's random-walk treatment of multiple scattering by calculating multiple-scattering angles for individual smallangle collisions and rotating the electron spin polarization. In their method, $\Theta$ was set to $0.1 \mathrm{rad}$, again based on the insensitivity of their model to $\Theta$ around this value.

\section{PRESENT MODEL}

\section{A. Introduction}

In this work we make the following improvements.

(i) The electron scattering channels are not artificially separated into multiple or plural scattering, but are considered integrally at each collision point.

(ii) We follow individual electrons and do not generate probabilities [cf. Eq. (9)] while the electron is still in the foil. Instead of detection probabilities, we generate electron detection events.
TABLE I. Summary of theoretical [19] angle-averaged Sherman function values for gold for various $E$ values, with detectors centered about $\theta=120^{\circ}$ and subtending half-cone angles of $\pm \Delta \theta$ at the source.

\begin{tabular}{ccccc}
\hline \hline & \multicolumn{4}{c}{$\Delta \theta$} \\
\cline { 2 - 5 }$E(\mathrm{keV})$ & $0^{\circ}$ & $5^{\circ}$ & $10^{\circ}$ & $20^{\circ}$ \\
\hline 10 & -0.262 & -0.260 & -0.254 & -0.228 \\
20 & -0.299 & -0.298 & -0.294 & -0.277 \\
40 & -0.338 & -0.337 & -0.334 & -0.319 \\
60 & -0.363 & -0.362 & -0.359 & -0.344 \\
90 & -0.389 & -0.387 & -0.383 & -0.368 \\
100 & -0.395 & -0.394 & -0.390 & -0.374 \\
120 & -0.405 & -0.403 & -0.399 & -0.383 \\
\hline \hline
\end{tabular}

(iii) We consider all elastic and inelastic scattering processes, e.g., we consider inelastic scattering using doubly differential cross sections derived from the Born approximation. For the predominantly small-angle inelastic scattering with energy loss $\left(E_{L}\right)$ below $1 \mathrm{keV}$, our model assumes a transformation of $\mathbf{P}$ equal to that for elastic scattering at the same angle and incident energy [cf. Eq. (6)]. This is a reasonable approximation for small-scattering-angle, small- $E_{L}$ processes since the projectile electron experiences an atomic potential similar to that for elastic scattering. For $E_{L}$ $>1 \mathrm{keV}$, the electrons scatter into a "black hole" and are lost. This procedure is discussed in more detail later on.

(iv) The improved screened-Coulomb data of Ross and Fink [19] for the elastic $S, T, U$, and $I$ parameters are used. Table I gives a summary of the calculated angle-averaged Sherman functions $S\left(E, \theta=120^{\circ}, \Delta \theta\right)$ for detectors centered at $\theta= \pm 120^{\circ}$ with opening half angles of $\Delta \theta$. We note that $S\left(E, \theta=120^{\circ}, \Delta \theta\right)$ equals $A_{\text {true }}$ when $P_{n}=1$.

\section{B. Method}

The present method makes extensive use of numerical algorithms in Ref. [20] and the random-number generator program RAN2 [21] which was tested in preliminary studies by us to have a periodicity exceeding $2.3 \times 10^{18}$ events. It was thus adequate for this work, which uses less than $10^{16}$ events. Since the total mean free path $\left(\lambda_{t}\right.$; Table II) of the electron at these $E$ values is longer than the diameter of a gold atom $(\approx 2.4 \AA[22])$, any crystal structure of the gold may be neglected. Given this assumption, the method consists of initializing the velocity and polarization $\left(P_{n}=1\right)$ of the incident electron to the desired values and then iterating the sequence described in the flowchart shown in Fig. 3.

The Monte Carlo program is started by downloading the appropriate $I, S, T$, and $U$ parameters, total elastic and inelastic cross sections, black-hole cross section, and other initial numerical parameters. The electron is initiated with $P_{n}=1$ and is fired along the $z$ direction (Fig. 1). The range of the electron is calculated using a Monte Carlo statistical weighting that decreases exponentially with the product of the distance traveled and the total scattering cross section. The electron can scatter in the foil through one of the three scattering processes: (i) elastic, (ii) inelastic and detectable (with the 
TABLE II. Total elastic cross sections $\left(\sigma_{e}\right)$, elastic mean free paths $\left(\lambda_{e}\right)$, ratios of total inelastic cross sections $\left(\sigma_{i}\right)$ to total elastic cross sections, and total scattering mean free paths $\left(\lambda_{t}=1 / n\left[\sigma_{e}+\sigma_{i}\right]\right)$ used in this work, at various $E$ values. The $\lambda_{t}$ were calculated using the $\sigma_{e}$ of Ross and Fink [19] and the $\sigma_{i}$ from the ratio $\sigma_{i} / \sigma_{e}$ of Misell [24] multiplied by $\sigma_{e}$ of Ross and Fink [19]. See text for details. Values in italics are interpolated.

\begin{tabular}{cccccccc}
\hline \hline & \multicolumn{2}{c}{ Reference [19] } & & \multicolumn{3}{c}{ Reference [24] } \\
\cline { 2 - 3 } \cline { 6 - 7 }$E(\mathrm{keV})$ & $\sigma_{e}\left(\AA^{2}\right)$ & $\lambda_{e}(\AA)$ & & $\sigma_{e}\left(\AA^{2}\right)$ & $\lambda_{e}\left(\AA^{2}\right)$ & $\sigma_{i} / \sigma_{e}$ & $\lambda_{t}(\AA)$ \\
\hline 10 & 0.807 & 21 & & 2.10 & 8 & 0.080 & 19 \\
20 & 0.545 & 31 & & 1.10 & 15 & 0.082 & 29 \\
30 & 0.424 & 40 & & 0.77 & 22 & 0.085 & 37 \\
40 & 0.361 & 47 & & 0.61 & 28 & 0.087 & 44 \\
60 & 0.281 & 60 & & 0.42 & 40 & 0.090 & 56 \\
80 & 0.240 & 71 & & 0.34 & 50 & 0.093 & 65 \\
90 & 0.219 & 77 & & 0.31 & 53 & 0.094 & 71 \\
100 & 0.203 & 83 & & 0.29 & 59 & 0.095 & 77 \\
120 & 0.185 & 92 & 0.27 & 61 & 0.096 & 85 \\
\hline \hline
\end{tabular}

ability to overcome detector retardation potential), or (iii) inelastic and undetectable (black-hole). The probability for this choice is based on the ratio of total cross sections for these processes.

If the electron is elastically scattered, the value of $\theta$ is determined from a weighting given by $I(\theta)$ whereas $\phi$ is determined from the term $\left[1-S(\theta) P_{n} \sin \phi\right]$ in Eq. (5). The polarization of the electron is then rotated [Eq. (6)] based on the values of $\theta$ and $\phi$, which determine the scattering plane in the coordinate frame of the scattering center. If the process is inelastic and the cumulative energy loss suffered by the electron is less than the detector retarding potential $\varepsilon$, the electron is deemed detectable and allowed to scatter further. Otherwise, it is terminated (black-hole) and a new electron trajectory is initiated. In this work, we consider values of $\varepsilon$ $\leqslant 1 \mathrm{keV}$ corresponding to most experimental situations. For an inelastically scattered electron, the energy loss value is selected according to the inelastic total cross section per unit energy loss, $d \sigma / d E_{L}$. The inelastically scattered electron is differentially scattered in $\theta$ and (random) $\phi$ based on the differential inelastic cross section. The total electron polarization $\mathbf{P}$ is transformed and the electron's energy is reduced by $E_{L}$.

Due to memory constraints, not all electron scattering angles were recorded. We economized in this by using one memory bin to count all electrons that were forward scattered (leaving the foil by the back face), one memory bin for all electrons that were backscattered (leaving the foil by the front face), and a number of memory bins representing concentric circular ring detectors centered about $\theta=120^{\circ}$ and $\phi=0^{\circ}, 180^{\circ}$, subtending half-cone angles $(\Delta \theta)$ of $10^{\circ}$ and $20^{\circ}$ at the collision region (respectively 0.098 and $0.42 \mathrm{sr}$ ). The sum of forward- and backscattered electrons constitutes a measure of the relative number of incident electrons. As discussed below, we made one set of runs with $\Delta \theta=5^{\circ}$ to more closely match the experimental conditions of Gay et al. [4].

We can greatly increase the efficiency of our calculations by considering several "virtual" foils of varying thickness at the same time ("stacked foil" method). We treat one foil as if it were one of the maximum thickness desired, but keep track of the maximum depth of any given trajectory along $z$. If an electron leaves the upstream foil surface, we look at its ultimate penetration depth. We then bin that scattered electron for each of the foils in the stack with thickness greater than the ultimate penetration depth of the scattered electron. We thus obtain better statistics for thicker foils, so we compensate by periodically adjusting the set of stacked foils so that the foil with the least counts becomes the thickest in the stack. We then continue the Monte Carlo calculation. Over several tens of cycles this method achieves comparable statistics for all stacked foils.

The stacked-foil algorithm has a major disadvantage in that the statistics of stacked foils are correlated. Consequently, in calculating errors incurred in parameters derived from least-squares fits to our stacked-foil data, we take the average statistical error for a single foil as representative of the incurred statistical error. This issue will be discussed further below.

Another advantage of our model is the addition of a black-hole $(\mathrm{BH})$ cross section, defined as the portion of the total cross section that prevents the electrons from ultimately surmounting the retarding-field grid in front of the electron detectors and being counted. The size of the BH cross section will thus depend on the energy loss required to keep an electron from being detected.

\section{Data used}

\section{Elastic scattering}

The elastic scattering data used here were obtained from the screened-Coulomb calculations of Ross and Fink [19], which, in the form of $S, T, U$, and $I$ parameters, were used to evaluate the spin-dependent elastic scattering processes [Eqs. (4)-(6)].

\section{Inelastic scattering}

To our knowledge, there exist no quantitative doubly differential cross sections (DDCS's) for inelastic electron scat- 


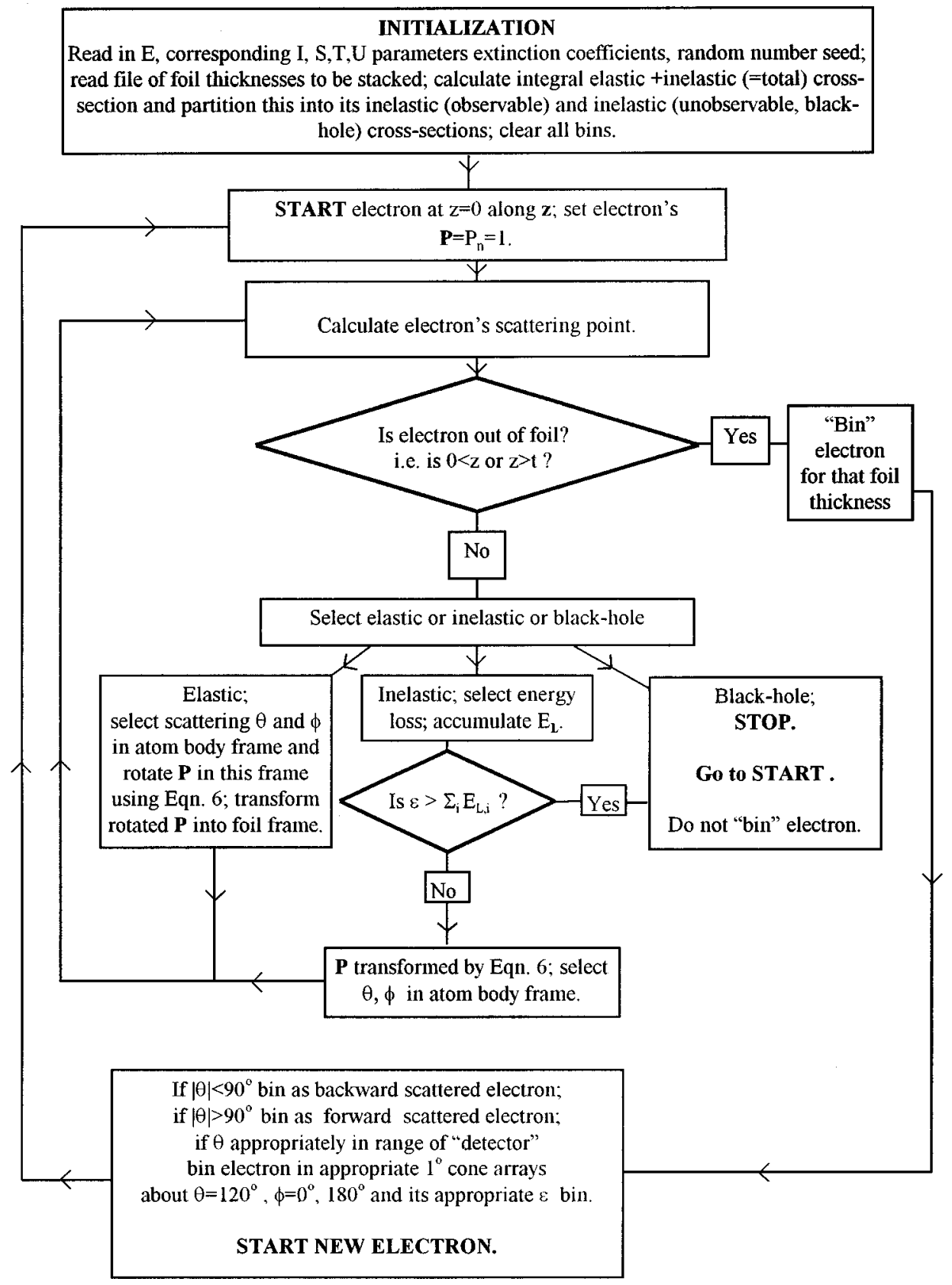

FIG. 3. Flowchart diagram for the Monte Carlo calculation (see text). The flowchart outlines the general route followed for a single foil thickness with elastic, inelastic, and "black-hole" channels. $E_{L, i}$ equals $E_{L}$ for the $i$ th scattering in any given electron trajectory $(i=1,2, \ldots)$.

tering from gold in the electron energy range considered here. However, it is possible to compute approximate highenergy inelastic scattering cross sections using Born approximation calculations based on optical data, e.g., oscillator strengths or extinction coefficients [23]. The Born approximation is considered reasonable for small-angle scattering at these electron energies. Since total elastic scattering cross sections are generally about an order of magnitude larger than total inelastic cross sections [24,25] over the range of $E$ used in this work (see Table II) and since inelastic processes are more strongly forward peaked than elastic ones [25], one should expect multiple scattering to produce a preponderance of inelastically scattered electrons in the forward direction.

In this work, we have derived the DDCS's from the Born approximation based on optical extinction coefficients. We start from the equation that relates the oscillator strength $f$ for an emission line to the photoabsorption cross section $\sigma_{p}(\omega)$ at the angular frequency $\omega$, as [26]

$$
\int \sigma_{p}(\omega) d \omega=2 \pi^{2} r_{0} c f
$$

where $r_{0}$ is the classical radius of the electron, and the integration is conducted over the line profile. For a continuous spectrum the differential form of Eq. (10) is applicable, i.e.,

$$
\sigma_{p}(\omega)=2 \pi^{2} r_{0} c \frac{d f}{d \omega}
$$




$$
\sigma_{p}(\omega)=\pi h r_{0} c \frac{d f}{d E_{L}} .
$$

One can now write the photoabsorption cross section $\sigma_{p}(\omega)$ in terms of the extinction coefficient $\kappa_{p}(\omega)[26,27]$, the number density of gold atoms $n$, and the wavelength $\lambda_{1}$, as

$$
\sigma_{p}(\omega)=\frac{4 \pi}{n \lambda_{1}} \kappa_{p}(\omega) .
$$

This yields

$$
\sigma_{p}(\omega)=3.44 \frac{d f}{d E_{L}}
$$

in units of $\AA^{2}$, where we substitute the value of $0.05907 \AA^{-3}$ for the value of $n$ for gold [22] (at $20^{\circ} \mathrm{C}$ ) in Eq. (13). Using Eqs. (13) and (14), we get

$$
\frac{d f}{d E_{L}}=56.33 \frac{\kappa_{p}(\omega)}{\lambda_{1}},
$$

where $\lambda_{1}$ is in angstroms. We can write Eq. (15) in terms of $E_{L}(\mathrm{eV})=h \omega / 2 \pi e(e$ is $1 \mathrm{esu})$ as

$$
\frac{d f}{d E_{L}}=4.978 \times 10^{-3} \kappa_{p}\left(E_{L}\right) E_{L}
$$

The theory of generalized oscillator strengths gives [23]

$$
\frac{d f}{d E_{L}}=\frac{E_{L}}{54.4} K^{2} \sqrt{E /\left(E-E_{L}\right)} \frac{d^{2} \sigma}{d \Omega d E_{L}},
$$

which means we can get the DDCS in terms of $d f / d E_{L}$ as

$$
\frac{d^{2} \sigma}{d \Omega d E_{L}}=0.271 \sqrt{\left(E-E_{L}\right) / E} \frac{\kappa_{p}\left(E_{L}\right)}{K^{2}},
$$

with $d^{2} \sigma / d \Omega d E_{L}$ in $\AA^{2} \mathrm{sr}^{-1} \mathrm{eV}^{-1}$ and the momentum transfer $K$ in $\AA^{-1}$. The available values of extinction coefficients for gold [27] are accurate to about $10 \%$.

The inelastic total cross sections obtained by integrating Eq. (18) are then normalized to the elastic total cross sections of Ross and Fink [19] using the total inelastic to inelastic cross section ratios given by Misell [24]. The normalization factor for our Born approximation inelastic cross-sections ranged between 0.05 to 0.2 . The above procedure is based on the following precepts. (i) The absolute values of the scattering cross sections calculated by Ross and Fink should be very reliable. As expected, these calculations agree with numerous other calculations (e.g., Ref. [18]). (ii) The absolute elastic scattering cross section measurements of Misell are less reliable than the calculations of Ross and Fink, because such measurements are hard to place on an absolute scale. (iii) The relative inelastic to elastic ratio measurements in Misell should be significantly more accurate than their absolute counterparts, because such ratios (at these $E$ values) cancel out the apparatus-sensitive parameters mentioned in (ii).

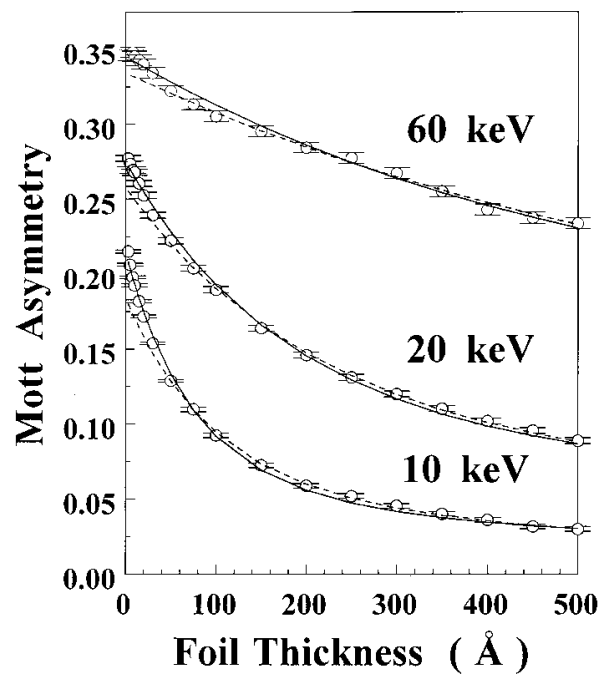

FIG. 4. $A$ vs $t$ for elastic scattering only. Data fitted with Eq. (19), solid line. Data fitted with Eq. (1), dashed line. Fits using Eq. (7) are almost identical to those of Eq. (19) and are therefore not shown. Table III contains a summary of parameters and $\chi_{\nu}^{2}$ values from fits to Eqs. (1), (7), and (19) (see text).

\section{DATA, ANALYSIS AND RESULTS}

\section{A. Elastic scattering only}

Figure 4 shows the dependence of $A$ on $t$ with $\Delta \theta$ $=20^{\circ}$, obtained from our model with elastic scattering only. In Fig. 4 we also show the present Monte Carlo data fitted to an exponential-type function [Eq. (1)] with $P_{n}=1$. A comparison of the same data is also made with an analytic form derived using the observations that (i) the differential asymmetry element $d A\left(t^{\prime}\right)$ contribution to the integral asymmetry $A$ from a planar foil element of thickness $d t^{\prime}$ located at a distance $t^{\prime}$ into the foil decreases with increasing $t^{\prime}$, approaching zero as $t^{\prime} \rightarrow \infty$; (ii) $d A\left(t^{\prime}\right) \rightarrow A_{\text {true }}$ as $t^{\prime} \rightarrow 0$; $A_{\text {true }}$ $=S(E, \theta, \Delta \theta)$ for $P_{n}=1$. Given these conditions, a reasonable guess is that $d A\left(t^{\prime}\right) \approx A_{\text {true }} \exp \left(-\xi t^{\prime}\right)$. We also assume that the efficiency for detecting electrons a distance $t^{\prime}$ into the foil follows an absorption law, i.e., is proportional to $\exp \left(-\alpha t^{\prime}\right)$, with $\alpha$ being a characteristic inverse length controlling the elastic scattering attenuation of electrons. We note that $\alpha$ is different from $\xi$ or $1 / \lambda_{e}\left(=n \sigma_{e}\right)$; we cannot assume that $\xi$ or $\alpha$ equals $1 / \lambda_{e}$ since the reduction of $A$ with $t$ involves complicated differential scattering events, whereas the attenuation of electrons in the foil follows the straightforward absorption law. We now obtain a weighted asymmetry average by integrating over the foil thickness:

$$
\begin{gathered}
A(t)=\frac{\int_{0}^{t} A_{\text {true }} e^{-(\xi+\alpha) t^{\prime}} d t^{\prime}}{\int_{0}^{t} e^{-\alpha t^{\prime}} d t^{\prime}} \\
=\frac{A_{\text {true }} \alpha\left(1-e^{-(\xi+\alpha) t}\right)}{(\xi+\alpha)\left(1-e^{-\alpha t}\right)} .
\end{gathered}
$$

This form has three adjustable parameters like Eq. (1), but is somewhat clumsier. Nevertheless, it has a better physical ba- 
TABLE III. Comparison of the $\xi, \alpha$, and $b_{3}$ parameters obtained from fitting all the data for $A$ vs $t$ for elastic scattering with Eq. (1), Wegener's Eq. (7), and our Eq. (19) (labeled appropriately in bottom row). The numbers in square brackets correspond to the power of 10.

\begin{tabular}{|c|c|c|c|c|c|c|c|c|c|c|}
\hline$E(\mathrm{keV})$ & $A_{0}$ & $\xi$ & $\alpha$ & $\chi_{v}^{2}$ & $A_{0}$ & $b_{3}$ & $\chi_{\nu}^{2}$ & $b_{3}[16]$ & $A_{0}$ & $\chi_{\nu}^{2}$ \\
\hline 10 & $0.215 \pm 0.002$ & $2.31 \pm 0.24[-3]$ & $2.15 \pm 0.07[-2]$ & 5.6 & $0.219 \pm 0.002$ & $1.32 \pm 0.03[-2]$ & 3.08 & & $0.184 \pm 0.003$ & 32.4 \\
\hline 20 & $0.268 \pm 0.002$ & $1.91 \pm 0.17[-3]$ & $7.87 \pm 0.23[-3]$ & 5.8 & $0.269 \pm 0.002$ & $4.29 \pm 0.01[-3]$ & 2.08 & & $0.258 \pm 0.003$ & 21.7 \\
\hline 40 & $0.312 \pm 0.002$ & $1.49 \pm 0.19[-3]$ & $3.04 \pm 0.10[-3]$ & 2.1 & $0.312 \pm 0.002$ & $1.52 \pm 0.03[-3]$ & 6.8 & & $0.299 \pm 0.002$ & 16.5 \\
\hline 46 & & & & & & & & $1.75 \pm 0.11[-3]$ & & \\
\hline 60 & $0.345 \pm 0.002$ & $1.73 \pm 0.22[-3]$ & $2.07 \pm 0.08[-3]$ & 2.4 & $0.343 \pm 0.002$ & $9.36 \pm 0.24[-4]$ & 4.7 & & $0.334 \pm 0.002$ & 11.3 \\
\hline 63 & & & & & & & & $1.24 \pm 0.07[-3]$ & & \\
\hline 90 & $0.365 \pm 0.001$ & $1.16 \pm 0.31[-3]$ & $1.46 \pm 0.25[-3]$ & 1.3 & $0.363 \pm 0.001$ & $5.14 \pm 0.12[-4]$ & 2.6 & & $0.356 \pm 0.002$ & 6.3 \\
\hline 100 & $0.379 \pm 0.001$ & $9.20 \pm 0.45[-4]$ & $1.35 \pm 0.28[-3]$ & 0.8 & $0.377 \pm 0.001$ & $3.87 \pm 0.10[-4]$ & 1.3 & $6.94 \pm 0.48[-4]$ & $0.375 \pm 0.001$ & 2.2 \\
\hline 120 & $0.382 \pm 0.001$ & $8.70 \pm 0.41[-4]$ & $1.25 \pm 0.25[-3]$ & 0.8 & $0.382 \pm 0.001$ & $3.15 \pm 0.09[-4]$ & 1.1 & & $0.382 \pm 0.001$ & 1.6 \\
\hline 133 & & & & & & & & $4.17 \pm 0.33[-4]$ & & \\
\hline 170 & & & & & & & & $3.19 \pm 0.23[-4]$ & & \\
\hline 204 & & & & & & & & $2.34 \pm 0.19[-4]$ & & \\
\hline 245 & & & & & & & & $1.74 \pm 0.16[-4]$ & & \\
\hline 290 & & & & & & & & $1.16 \pm 0.10[-4]$ & & \\
\hline Eq. & (19) & (19) & (19) & (19) & (7) & (7) & (7) & (7) & (1) & (1) \\
\hline
\end{tabular}

sis than Eq. (1). Foil-thickness extrapolations based on Eq. (1) are discussed by, e.g., Gay et al. [4] and Gellrich and Kessler [2].

The results of the Fig. 4 fits using Eqs. (1), (7), and (19) are summarized in Table III. The errors in the fitting parameters include the average statistical uncertainty (one standard deviation) for a single foil in the stack of foils combined in quadrature with the fitting error from the nonlinear leastsquares program which assumes equal error for all the foils used (one standard deviation). In the stacked-foil method, each foil has comparable statistics (see Sec. IVB). This method of error estimation should lead to conservative errors, since uncertainty from only a single foil is considered, and not the combined reduced uncertainty of the full stack of foils. The values of $\chi_{\nu}^{2}$ are obtained using the statistical errors of each foil. In Sec. V B 1 we address the validity of our stacked-foil data when compared to single-foil data.

To check this error estimation method, all data points $A(t)$ were allowed to randomly vary about the normal distribution given by their standard deviation statistical uncertainty $\pm \Delta A(t)$, and the new set of $A(t)$ values were fitted to the relevant extrapolation equation (1), (7), or (19). For approximately 30 such sets of data, the variation (standard deviation) of the extrapolated $A_{0}[=A(t=0)]$ values or those of the other coefficients in Eq. (1), (7), or (19) [e.g., $b_{3}$ in Eq. (7)] compared satisfactorily with our error determination above. In all cases the second method gave standard deviation errors that averaged 20-30\% lower than our method.

From Table III, it is clear that the fits using Eqs. (7) and (19) are both reasonable, but (based on the $\chi_{\nu}^{2}$ values) qualitative, especially at low $E$ values. These fits give extrapolated $A_{0}$ values significantly different from $S(E, \theta, \Delta \theta)$ ( $=A_{\text {true }}$ ) of Table I, i.e., outside the sub-1\% error bars of the data for $E<60 \mathrm{keV}$. The $\chi_{\nu}^{2}$ values indicate that the fits using Eq. (19) are somewhat better than those of Eq. (7) as one would expect, given the extra degree of freedom in Eq. (19).
The coefficients $\xi$ and $\alpha$ determined from fits using Eq. (19) are shown in Table III together with the $b_{3}$ values from fitting Eq. (7) to these data. Equation (1) gave worse fits to these data than did Eqs. (7) and (19).

In Fig. 5 we compare our model's $b_{3}$ values [Eq. (7)] with those quoted by Hnizdo [16]. Since Eq. (7) is derived considering only single and double scattering, its validity is limited roughly to thicknesses such that $\lambda_{e} t<1$ (Table II). At $E=10 \mathrm{keV}$ this corresponds to $t<20 \AA$, whereas at $E$ $=100 \mathrm{keV}$ the approximate range of validity extends to 100 $\AA$. The $b_{3}$ values of Hnizdo are higher than ours at all $E$ values by about $20 \%$. This is not due to differences in the $S$, $T, U, I$ parameters, which differ above $40 \mathrm{keV}$ by less than $1 \%$. The larger $b_{3}$ values obtained by Hnizdo correspond to a more rapid asymmetry reduction in the foil [see Eq. (7)]. This is possibly the result of the fact that in Hnizdo's model a multiple scattering always precedes plural scattering. This bias attached to multiple scattering may allow the electron to depolarize more quickly and may be a larger effect than an-

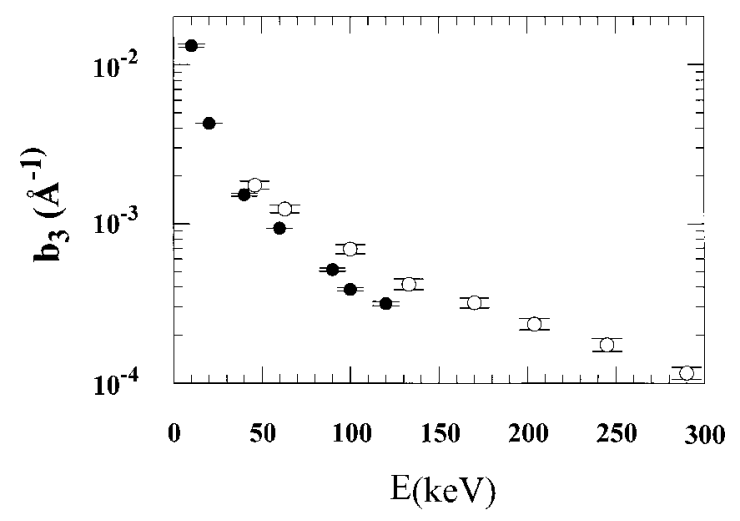

FIG. 5. Comparison of $b_{3}$ values derived from fitting Eq. (7) to our elastic scattering data $(\bigcirc)$ and the $b_{3}$ values of Hnizdo [16] ( $\left.\bigcirc\right)$. See also Table III and discussion in the text. 


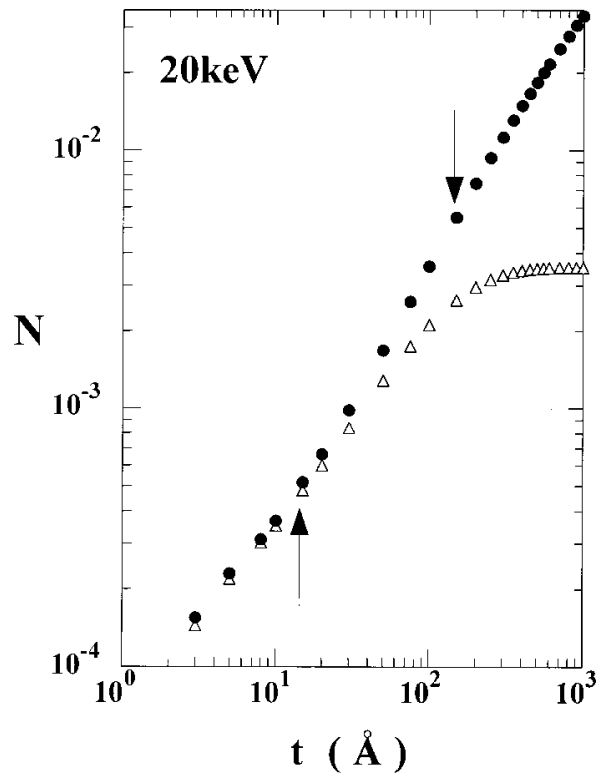

FIG. 6. $N$ vs $t$ for elastic scattering only (-) and elastic plus inelastic black-hole scattering $(\triangle)$ for $E=20 \mathrm{keV}$. The points of inflection (indicated by arrows) occur at $t=140 \pm 5$ and 13 $\pm 2.5 \AA$, respectively. The $\Delta \theta$ of the detectors is $20^{\circ}$.

ticipated. This conclusion was also reached by Qiao and Kakizaki [17]. Moreover, Hnizdo's method allows the electron to travel farther through the foil. By deflecting the electron through a cumulative multiple-scattering angle the electron trajectories are elongated by a factor of one divided by the cosine of the accumulated scattering angle. The increased trajectory length will produce lower $A$ values.

As a point of interest, we investigated $A$ vs $t$ at $E=10,20$, and $40 \mathrm{keV}$ when the cross section for elastic small-angle (multiple) scattering $\left(\theta<20^{\circ}\right)$ was set to zero. We found that the reduction of $A$ due to forward (multiple) scattering is rapid in the first few tens of angstroms of the foil. For larger $t$ the normal $A$ values remain about $15 \%$ below the $A$ values with the reduced multiple scattering.

\section{B. Elastic plus inelastic scattering}

\section{Black-hole treatment of inelastic scattering}

We now turn to the case where inelastic processes occur, but only elastically scattered electrons are observed. Thus the role of inelastic electron scattering processes is considered without having to account for the energies of the unobserved electrons (black-hole events). Figure 6 shows the detected count rate $N$ as a function of $t$ for elastic scattering only, and for elastic plus black-hole scattering. Both curves show an approximately exponential increase of $N$ with respect to $t$ for small $t$. This is followed by an inflection point in both cases. This inflection characteristic has also been observed experimentally by Gay et al. [4]. The inflection point in $N$ vs $t$ is consistent with a dominance of inelastic over elastic scattering for thin foils for the black-hole algorithm. In the case of elastic scattering only, the infinite- thickness behavior of $N$ vs $t$ must still be asymptotic, which requires an inflection point.

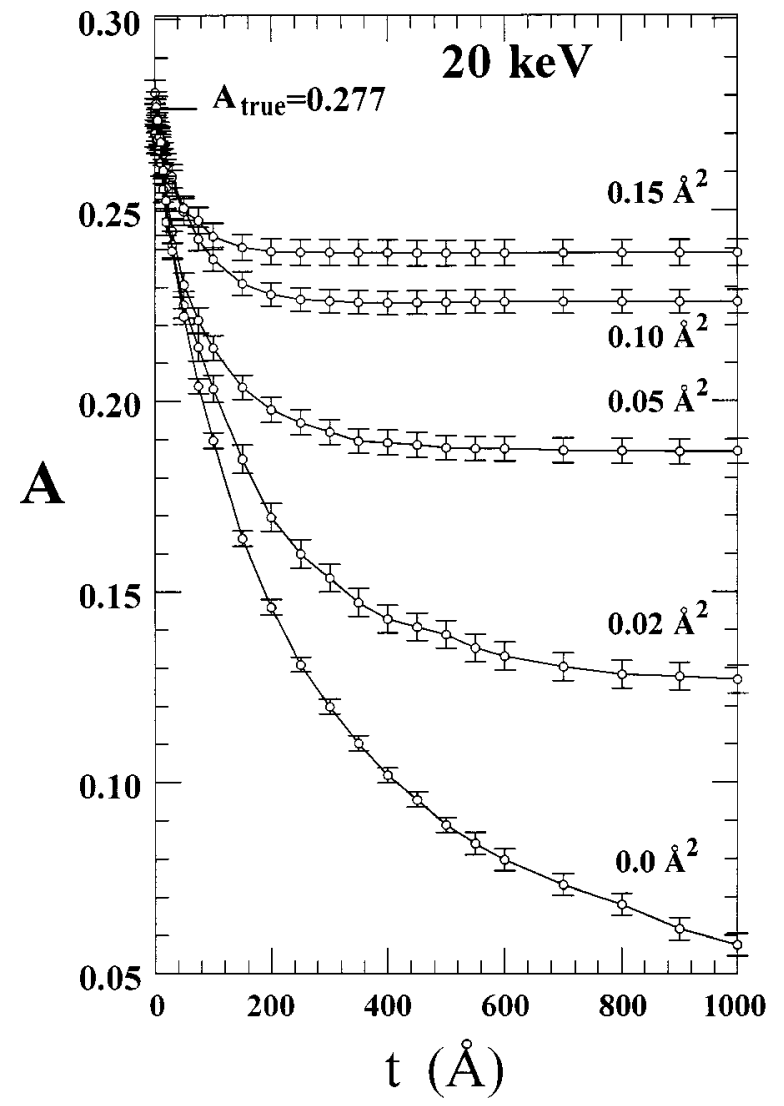

FIG. 7. $A$ vs $t$ plots for elastic plus black-hole scattering at $E$ $=20 \mathrm{keV}$, for various $\mathrm{BH}$ cross sections $\left(\AA^{2}\right)$ and $\varepsilon=0$. The $\Delta \theta$ of the detectors is $20^{\circ}$. The error bars are the statistical errors, and are approximately the same for each foil because of our stacked-foil algorithm (see text). The solid lines are drawn to guide the eye.

Figure 7 demonstrates the strong effect on $A$ of increasing the $\mathrm{BH}$ cross section in our model at $E=20 \mathrm{keV}$. As the black-hole cross section increases, fewer and fewer electrons that have undergone depolarizing plural scattering are detected. In effect, only electrons scattered from an increasingly thin surface layer contribute to the measured asymmetry, resulting in higher asymptotic values of $A$ as $t \rightarrow \infty$. This asymptotic behavior is evident in $N$ as well, and is shown in Fig. 8. At higher $E$, the total elastic cross section falls rapidly and $A$ does not reach a saturated value for the range of thickness we consider here.

In Fig. 8 we also compare the results of runs in which we used single foils of varying thickness as opposed to the more efficient stacked-foil method. Our single-foil results show excellent agreement with the stacked-foil data and confirm our expectation that the stacked-foil method should give results equivalent to those obtained with single foils. This observation also provides support for the method of error estimation used in analysis of the stacked-foil results (see Sec. V B 2).

Using total elastic to total inelastic cross-section ratios from Ref. [24] (see Table II) and the total elastic cross sections of Ref. [19], we calculated the integrated $\mathrm{BH}$ cross sections. For example, at $E=20 \mathrm{keV}$ we used 0.082 of the elastic cross section $\left(0.545 \AA^{2}\right)$ to give an inelastic cross 


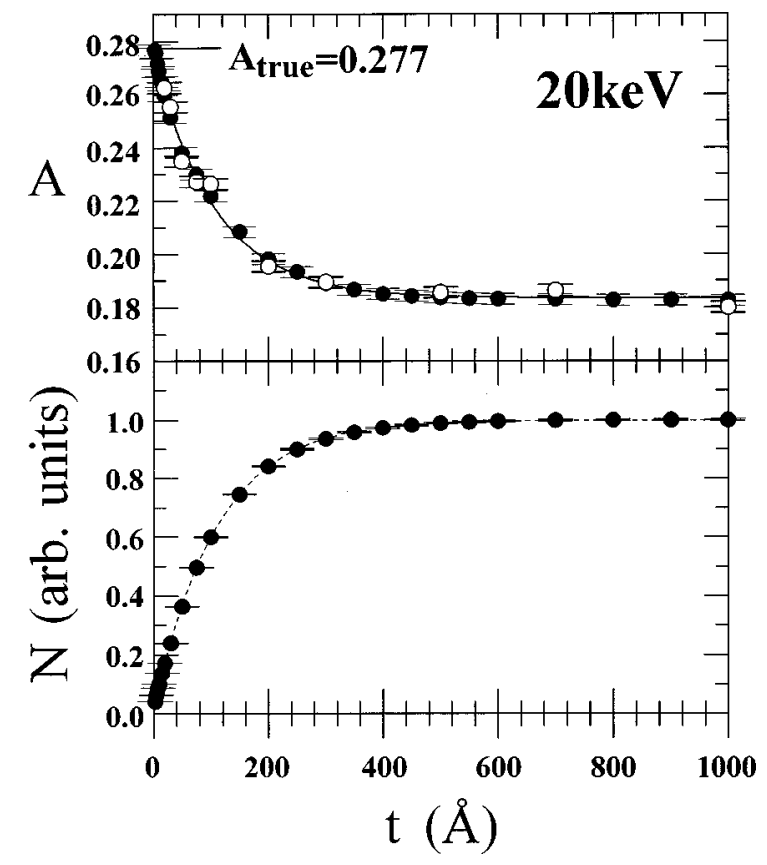

FIG. 8. Graphs showing behavior of $A$ (upper graph) and $N$ (lower graph) as functions of $t$ at $E=20 \mathrm{keV}$. The BH cross section is $0.0449 \AA^{2}$ (=0.082 of the elastic cross section). The $\Delta \theta$ of the detectors is $20^{\circ}$. Legend: $\bigcirc$, stacked foils; $\bigcirc$, single foils. The solid line is a fit to Eq. (1) for the single-foil data (minimum foil thickness is $20 \AA$ ); see text. The dashed line is a fit using the form of Eq. (21).

section of $0.0449 \AA^{2}$ (see also Table II). We compare our BH model (with $\Delta \theta=20^{\circ}$ ) to the experimental work of Gay et al. [4] and Uhrig et al. [28] in Fig. 9 for all $E$ values used in the experiment. Excellent agreement is found between experiment and our model considering that we hold fixed the BH cross section, our most sensitive parameter. However, there remain some disagreements between our model and the experimental data at larger $t$ values, especially for those data that have very small error bars, e.g., for $t=682 \AA$ at $E=20$ and $60 \mathrm{keV}$. This problem may be model related since we have been forced to use large solid angles for our detectors in order to acquire adequate statistics. However, a large part of the disagreement appears to be due to the experimental data. For example, at $E=20 \mathrm{keV}$ and $t=682 \AA$, the $A$ value from experiment is larger than its value at $341 \AA$, which is not physical. In our comparison with the data of Gay et al. [4] we have normalized their data to our theory at their smallest foil thickness of $34 \AA$. We note that at 40 and $100 \mathrm{keV}$ agreement between our model and experiment is excellent over the entire range of foil thickness. We have normalized the data of Uhrig et al. to ours at $t \geqslant 400 \AA$, because of the excellent agreement between their data and ours in this range of $t$. However, their experimental data do not exhibit the nonlinear rise in $A$ indicated by our model for $t<400 \AA$. This deviation of experiment from our model is not presently explainable, and it is also in disagreement with the experimental data of Gay et al. [4].

It is important to note that in the above comparisons our $\Delta \theta=20^{\circ}$ differs from the experimental values of $\Delta \theta \approx 0.5^{\circ}$

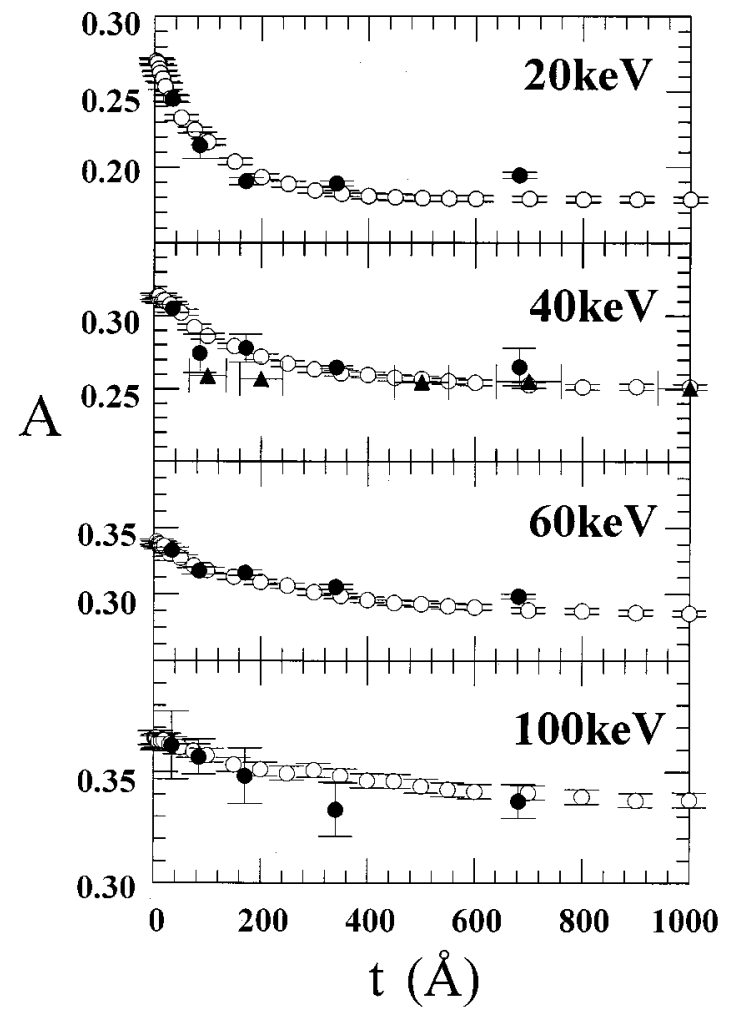

FIG. 9. $A$ vs $t$ for elastically scattered electrons $(\varepsilon=0)$ for various $E$ values (black-hole model). Legend: $\bigcirc$, present work; $\bullet$, experiment of Gay et al. [4] $(\varepsilon=4 \mathrm{eV}) ; \boldsymbol{\Delta}$, experiment of Uhrig et al. $[28](\varepsilon \approx 0 \mathrm{eV})$. See text.

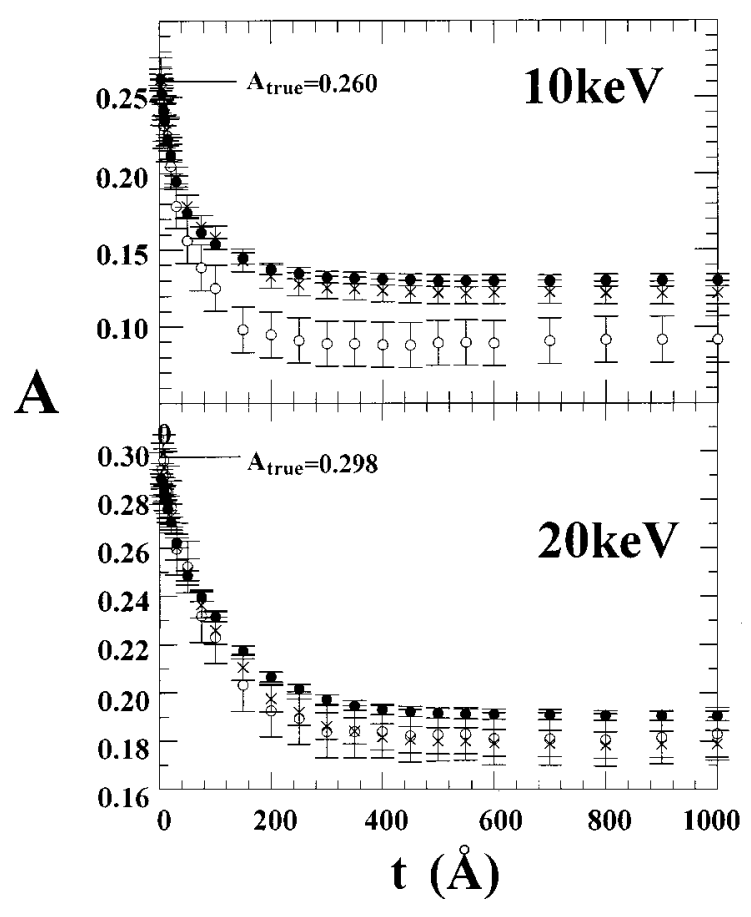

FIG. 10. Variation of $A$ with detector $\Delta \theta$, for $E=10$ and $20 \mathrm{keV}$. Legend: $\bigcirc, \Delta \theta=5^{\circ} ; \times, \Delta \theta=10^{\circ}$; and $\bigcirc, \Delta \theta=20^{\circ}$. The $A$ values for the different $\Delta \theta$ data are normalized to the $\Delta \theta=5^{\circ}$ data at $t=3 \AA$ to highlight the relative shapes of these curves (see text). 
TABLE IV. Fitting statistics for the present BH model for $A$ vs $t$ using Eq. (1) and (19) for different $E, \Delta \theta$, and minimum foil thicknesses. The minimum foil thickness used in the fit is given in the topmost row in parentheses. Equation (21) is used to fit the $N$ vs $t$ data to obtain $\Gamma^{\prime}$. The maximum foil thickness used is $1000 \AA$. The $A_{\text {true }}$ values $\left(P_{n}=1\right)$ are taken from Table I. The numbers in parentheses are errors in the least significant digits.

\begin{tabular}{cccccccccccccccc}
\hline \hline$E(\mathrm{keV})$ & $\Delta \theta(\mathrm{deg})$ & $A_{\text {true }}$ & $A_{0}(3 \AA)$ & $\chi_{\nu}^{2}$ & $A_{0}(3 \AA)$ & $\chi_{\nu}^{2}$ & $\Gamma(3 \AA)\left(\AA^{-1}\right)$ & $\Gamma^{\prime}(3 \AA)\left(\AA^{-1}\right)$ & $A_{0}(30 \AA)$ & $\chi_{v}^{2}$ & $A_{0}(50 \AA)$ & $\chi_{\nu}^{2}$ & $A_{0}(200 \AA)$ & $\chi_{\nu}^{2}$ \\
\hline 10 & 10 & 0.254 & $0.249(4)$ & 0.15 & $0.249(5)$ & 0.18 & $0.0168(12)$ & $0.0137(4)$ & $0.219(9)$ & 0.16 & $0.213(11)$ & 0.37 & $0.203(5)$ & 0.20 \\
10 & 20 & 0.228 & $0.224(4)$ & 0.19 & $0.223(4)$ & 0.23 & $0.0219(12)$ & $0.0139(4)$ & $0.190(7)$ & 0.11 & $0.175(7)$ & 0.27 & $0.170(4)$ & 0.23 \\
20 & 10 & 0.294 & $0.291(2)$ & 0.22 & $0.290(2)$ & 0.26 & $0.0088(4)$ & $0.0091(2)$ & $0.289(7)$ & 0.16 & $0.283(5)$ & 0.34 & $0.269(3)$ & 0.39 \\
20 & 20 & 0.277 & $0.275(2)$ & 0.17 & $0.274(2)$ & 0.17 & $0.0094(4)$ & $0.0090(1)$ & $0.264(4)$ & 0.13 & $0.262(4)$ & 0.30 & $0.260(2)$ & 0.33 \\
40 & 10 & 0.334 & $0.332(2)$ & 0.20 & $0.331(2)$ & 0.24 & $0.0052(3)$ & $0.0060(3)$ & $0.328(2)$ & 0.23 & $0.324(3)$ & 0.27 & $0.323(2)$ & 0.30 \\
40 & 20 & 0.319 & $0.316(2)$ & 0.33 & $0.317(2)$ & 0.35 & $0.0057(2)$ & $0.0060(1)$ & $0.312(4)$ & 0.31 & $0.310(4)$ & 0.33 & $0.310(2)$ & 0.37 \\
60 & 10 & 0.359 & $0.360(2)$ & 0.33 & $0.361(2)$ & 0.40 & $0.0039(3)$ & $0.0045(2)$ & $0.354(2)$ & 0.25 & $0.354(3)$ & 0.28 & $0.339(2)$ & 0.33 \\
60 & 20 & 0.344 & $0.344(2)$ & 0.30 & $0.344(2)$ & 0.36 & $0.0043(2)$ & $0.0047(1)$ & $0.339(2)$ & 0.17 & $0.339(2)$ & 0.27 & $0.336(2)$ & 0.30 \\
90 & 10 & 0.383 & $0.381(3)$ & 0.19 & $0.382(3)$ & 0.23 & $0.0034(5)$ & $0.0035(1)$ & $0.380(2)$ & 0.15 & $0.376(3)$ & 0.18 & $0.370(3)$ & 0.20 \\
90 & 20 & 0.368 & $0.369(2)$ & 0.15 & $0.369(2)$ & 0.19 & $0.0039(3)$ & $0.0038(1)$ & $0.365(3)$ & 0.10 & $0.364(3)$ & 0.19 & $0.360(2)$ & 0.23 \\
100 & 10 & 0.390 & $0.392(2)$ & 0.19 & $0.392(2)$ & 0.21 & $0.0027(2)$ & $0.0026(1)$ & $0.389(2)$ & 0.13 & $0.388(3)$ & 0.15 & $0.384(3)$ & 0.18 \\
100 & 20 & 0.374 & $0.375(2)$ & 0.11 & $0.375(2)$ & 0.13 & $0.0029(2)$ & $0.0027(1)$ & $0.373(2)$ & 0.10 & $0.373(2)$ & 0.12 & $0.367(3)$ & 0.14 \\
120 & 10 & 0.399 & $0.398(2)$ & 0.20 & $0.397(2)$ & 0.24 & $0.0019(2)$ & $0.0026(1)$ & $0.398(2)$ & 0.11 & $0.397(2)$ & 0.15 & $0.394(2)$ & 0.18 \\
120 & 20 & 0.383 & $0.381(2)$ & 0.22 & $0.382(2)$ & 0.23 & $0.0022(2)$ & $0.0028(1)$ & $0.383(2)$ & 0.14 & $0.383(2)$ & 0.15 & $0.377(2)$ & 0.17 \\
& Eq. & & $(1)$ & $(1)$ & $(19)$ & $(19)$ & $(1)$ & $(21)$ & $(1)$ & $(1)$ & $(1)$ & $(1)$ & $(1)$ & $(1)$ \\
\hline \hline
\end{tabular}

[4] or $\approx 5^{\circ}$ [28]. To justify using $\Delta \theta=20^{\circ}$ we show in Fig. 10 the extent to which $A$ is affected when $\Delta \theta$ is reduced at $E=10$ and $20 \mathrm{keV}$. (We do this at $E=10$ and $20 \mathrm{keV}$ because this is where the solid-angle effect should be most pronounced and our statistics are best.) We note that the relative change in $A$ between the $\Delta \theta=5^{\circ}$ and $20^{\circ}$ data at $E$ $=10 \mathrm{keV}$ is about $40 \%$ at $t=1000 \AA$, and significantly outside the two sets of error bars. This is the worst case. At $E$ $=20 \mathrm{keV}$ the maximum difference at large $t$ is reduced to $\approx 4 \%$. This makes comparison of our large- $\Delta \theta$ calculations at $20 \mathrm{keV}$ and above with experimental data reasonable. We note the larger error bars on the $\Delta \theta=5^{\circ}$ results due to poorer statistics.

\section{2. $\varepsilon=0$ foil thickness extrapolations}

Gay et al. [4] and Fletcher, Gay, and Lubell [5] discuss the various functional forms for extrapolation of $A$ to the single-atom scattering limit. In addition, Wegener $[12,13]$, Greenberg et al. [14], and Braicovich and de Michelis [15] have suggested the use of Eq. (7) and variants of it for extrapolations at high $E$ values. Several observations from our model that have important ramifications for such extrapolation methods are now summarized.

(a) Comparison between Wegener-type and exponentialtype forms. Fits using exponential forms [Eqs. (1) or (19)] to real data or our model data with inelastic scattering should be better than fits to pure elastic scattering model results. The attenuation of electrons by inelastic processes causes $A$ to reach a nonzero asymptotic value for large $t$ (Fig. 7), which is allowed in Eqs. (1) and (19). In Eq. (7), A tends to an asymptotic value of zero. Therefore, for the present purposes Eq. (7) is not applicable, even if a correction to it is made such as

$$
A(t)=\approx \frac{A^{\prime}}{1+b_{3}^{\prime}(E, \theta) t}+C
$$

with $A^{\prime}+C=A_{\text {true }}$. This is because the condition $d A(t)$ $\rightarrow 0$ as $t \rightarrow \infty$ [ see condition (ii) in Sec. V A] is not met.

(b) Comparison between exponential fits. In Table IV, we compare the extrapolated $A_{0}$, using Eqs. (1) and (19), with our $\Delta \theta=10^{\circ}$ and $20^{\circ}$ data using varying minimum foil thicknesses in the fit. We have already commented on the dependence of $A$ vs $t$ curves as a function of $\Delta \theta$. From the table, we observe that, for extrapolations to $t=0$ using a full range of foils, both Eqs. (1) and (19) yield $A_{\text {true }}$, within at most twice their fitting uncertainties. We note that the $\chi_{\nu}^{2}$ values for these fits are all well below 1 . However, these $\chi_{\nu}^{2}$ values are underestimates, since the asymmetries for the various stacked-foil thicknesses are correlated. As a check, single-foil data taken at $E=20 \mathrm{keV}$ and $\Delta \theta=20^{\circ}$ (minimum foil thickness $20 \AA$; see Fig. 8) fitted to Eq. (1) give a value of $A_{0}=0.276 \pm 0.005$ with a $\chi_{\nu}^{2}$ of 1.85 . With the same data using Eq. (19) we get a value of $A_{0}=0.277 \pm 0.005$ with $\chi_{\nu}^{2}$ $=2.25$. From this we conclude that Eq. (1) used in several past experiments [2-5] is adequate for extrapolation purposes.

(c) Foil-thickness-related extrapolations. Table V summarizes the results of extrapolating $A(t)$ and its reciprocal to $t$ $=0$ or $N=0$ using various fitting forms. From Table $\mathrm{V}$ we observe that exponential-type extrapolations are the most reliable. Both the exponential forms of $1 / A$ vs $t$ and $A$ vs $t$ give accuracies better than $1 \%$ when using foils in the complete range of 3 to $1000 \AA$. However, when we limit the thinnest foil to $30 \AA$, ${ }^{1}$ the situation worsens and sub-1\% extrapolations are reached only at $E \geqslant 60 \mathrm{keV}$. Our worst-case extrapolation at $10 \mathrm{keV}$ misses $A_{\text {true }}$ by about $2.5 \%$. Our model also indicates that both exponential $1 / A$ vs $t$ and exponential $A$ vs $t$ extrapolations are equivalent and perform essentially

\footnotetext{
${ }^{1}$ The $30 \AA$ limiting foil thickness is chosen here, since it is comparable to the thinnest foil used in [4].
} 
TABLE V. Extrapolated $A_{0}$ values from (column labels) $A, A(t)=a_{0}+b_{0} t ; B, A(t)=a_{1}+b_{1} e^{-\Gamma t} ; C$, $A(t)=a_{2}+b_{2} N(t) ; D, 1 / A(t)=a_{4}+b_{4} t ; E, 1 / A(t)=a_{5}+b_{5} e^{-\Gamma t} ; F, 1 / A(t)=a_{6}+b_{6} N(t)$, where the $a_{0-6}$, $b_{0-6}$ parameters are fitting constants. The upper part of the table is for foil thickness in the range of 30 to $1000 \AA$ and the lower part is for the range of 3-1000 $\AA$ with $\Delta \theta=20 \mathrm{deg}$. Numbers in parentheses are errors in the least significant digits. Numbers in italics are the $\chi_{\nu}^{2}$ values.

\begin{tabular}{|c|c|c|c|c|c|c|c|}
\hline$E(\mathrm{keV})$ & $A$ & $B$ & $C$ & $D$ & $E$ & $F$ & $A_{\text {true }}$ \\
\hline \multicolumn{8}{|c|}{ Fits using foils $30 \AA$ and greater } \\
\hline \multirow[t]{2}{*}{10} & $0.134(11)$ & $0.190(7)$ & $0.189(36)$ & $0.132(55)$ & $0.198(6)$ & $0.206(15)$ & 0.228 \\
\hline & 7 & 0.11 & 21 & 11 & 0.12 & 42 & \\
\hline \multirow[t]{2}{*}{20} & $0.216(12)$ & $0.264(4)$ & $0.258(4)$ & $0.215(13)$ & $0.261(4)$ & $0.288(4)$ & 0.277 \\
\hline & 8 & 0.13 & 13 & 42 & 0.35 & 9 & \\
\hline \multirow[t]{2}{*}{40} & $0.289(9)$ & $0.312(4)$ & $0.309(3)$ & $0.289(9)$ & $0.303(4)$ & $0.324(3)$ & 0.319 \\
\hline & 14 & 0.31 & 2.1 & 19 & 0.24 & 2.1 & \\
\hline \multirow[t]{2}{*}{60} & $0.320(6)$ & $0.339(2)$ & $0.339(3)$ & $0.320(5)$ & $0.337(2)$ & $0.341(3)$ & 0.344 \\
\hline & 8 & 0.17 & 0.85 & 6 & 0.11 & 0.82 & \\
\hline \multirow[t]{2}{*}{90} & $0.355(5)$ & $0.365(3)$ & $0.365(3)$ & $0.356(4)$ & $0.363(4)$ & $0.365(3)$ & 0.368 \\
\hline & 1.17 & 0.10 & 0.33 & 1.04 & 0.16 & 0.67 & \\
\hline \multirow[t]{2}{*}{100} & $0.364(3)$ & $0.373(2)$ & $0.375(2)$ & $0.365(2)$ & $0.371(2)$ & $0.376(2)$ & 0.374 \\
\hline & 0.22 & 0.41 & 0.19 & 0.54 & 0.10 & 0.54 & \\
\hline \multirow[t]{3}{*}{120} & $0.374(2)$ & $0.838(2)$ & $0.384(2)$ & $0.376(2)$ & $0.381(2)$ & $0.385(2)$ & 0.383 \\
\hline & 0.35 & 0.10 & 0.33 & 0.29 & 0.10 & 0.34 & \\
\hline & \multicolumn{7}{|c|}{ Fits using all foils } \\
\hline \multirow[t]{2}{*}{10} & $0.167(43)$ & $0.224(4)$ & $0.211(8)$ & $0.159(18)$ & $0.222(5)$ & $0.227(6)$ & 0.228 \\
\hline & 49 & 0.19 & 240 & 10 & 0.20 & 175 & \\
\hline \multirow[t]{2}{*}{20} & $0.240(13)$ & $0.275(2)$ & $0.271(2)$ & $0.237(18)$ & $0.273(3)$ & $0.285(5)$ & 0.277 \\
\hline & 33 & 0.17 & 19 & 13.00 & 0.22 & 24 & \\
\hline \multirow[t]{2}{*}{40} & $0.301(8)$ & $0.316(2)$ & $0.318(2)$ & $0.299(9)$ & $0.321(3)$ & $0.311(4)$ & 0.319 \\
\hline & 40 & 0.33 & 1.41 & 35.00 & 0.99 & 1.95 & \\
\hline \multirow[t]{2}{*}{60} & $0.328(7)$ & $0.344(2)$ & $0.343(2)$ & $0.331(6)$ & $0.341(3)$ & $0.342(3)$ & 0.344 \\
\hline & 19 & 0.30 & 0.61 & 13.70 & 0.34 & 0.82 & \\
\hline \multirow[t]{2}{*}{90} & $0.365(5)$ & $0.369(2)$ & $0.368(2)$ & $0.364(5)$ & $0.368(2)$ & $0.367(3)$ & 0.368 \\
\hline & 1.11 & 0.15 & 0.40 & 2.50 & 0.11 & 0.30 & \\
\hline \multirow[t]{2}{*}{100} & $0.372(2)$ & $0.375(2)$ & $0.376(3)$ & $0.371(2)$ & $0.374(2)$ & $0.372(2)$ & 0.374 \\
\hline & 0.405 & 0.11 & 0.37 & 0.762 & 0.10 & 0.87 & \\
\hline \multirow[t]{2}{*}{120} & $0.380(2)$ & $0.381(2)$ & $0.383(3)$ & $0.380(3)$ & $0.381(2)$ & $0.383(2)$ & 0.383 \\
\hline & 0.56 & 0.22 & 0.51 & 0.57 & 0.154 & 0.41 & \\
\hline
\end{tabular}

the same with regard to extrapolation precision and accuracy. Therefore for foil thickness extrapolations we again recommend exponential forms such as Eq. (1), as are also recommended by Gay et al. [4].

From Table $\mathrm{V}$ we see that linear extrapolations are precise in the sub-1\% region only at high $E \geqslant 100 \mathrm{keV}$ values. The quantitative results in Table $\mathrm{V}$ support the arguments put forward by Gay et al. [4] to explain the problems associated with determining $A_{\text {true }}$ at lower $E$. The results clearly show that it is not possible to linearly extrapolate properly to zero foil thickness at low $E$ values with a restricted set of foils. For example, in the measurements of Campbell et al. [8], whose minimum foil thickness was $130 \AA$, all linear extrapolations with $E<100 \mathrm{keV}$ will fall markedly below the $A_{\text {true }}$ value in a manner that is uncorrectable by simple, linear algorithms. With thin enough foils, one should be able to extrapolate accurately to $A_{\text {true }}$ even at $10 \mathrm{keV}$. But, one might ask, how thin is thin enough?

In Fig. 11 we summarize the deviations of our exponential
$A$ vs $t$ extrapolation from the $A_{\text {true }}$ values for several limited foil ranges (see also Table IV). At the lowest energies, it is apparent that extrapolations accurate to better than $1 \%$ are not obtainable even with the use of $3 \AA$ foils. At $120 \mathrm{keV}$, sub-1\% accuracy is possible even with the thinnest film being $\geqslant 50 \AA$ thick. These results are summarized further in Fig. 12, which shows the minimum foil thickness required to extrapolate to $A_{\text {true }}$ with accuracies of $1 \%$ and $2 \%$.

(d) N-type extrapolations. Since the yield of elastically scattered electrons is dependent on the absorption of electrons into inelastic channels, $N$ can be expected to behave similarly to an absorption-type formula of the form

$$
N(E, t, \theta, \varepsilon)=N(E, \infty, \theta, \varepsilon)\left\{1-\exp \left[-\Gamma^{\prime}(E, \theta, \varepsilon) t\right]\right\},
$$

which is very similar to the inverse of Eq. (1) with an energy loss parameter $\Gamma^{\prime}$ replacing the asymmetry reduction parameter $\Gamma$. It provides an excellent fit to the $N$ vs $t$ results from 


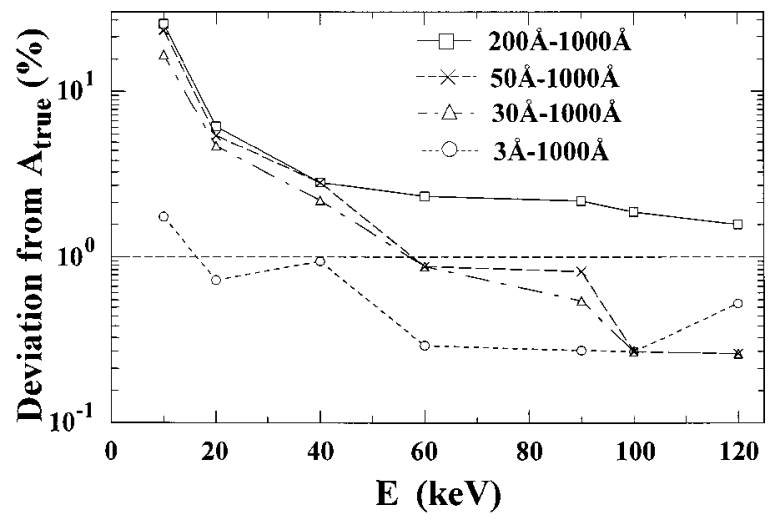

FIG. 11. Deviation of extrapolated values from $A_{\text {true }}$ for several minimum foil thicknesses ranging from 3 to $200 \AA$ as a function of $E$, using Eq. (1) to fit the data.

our model (Fig. 8). Such a behavior has been observed experimentally $[4,5]$. One might thus expect a linear dependence of $A(t)$ vs $N(t)$ based on the seemingly inverse behavior of $A$ vs $t$. However, our plots of $A(t, \varepsilon=0)$ vs $N(t, \varepsilon=0)$, shown in Fig. 13, are not linear, but show "oscillatory" behavior about a straight line fit which becomes more pronounced with decreasing $E$. These oscillations were not observed by Gay et al. [4], possibly because of their relatively small number of data points. This nonlinearity can be predicted from the data of Fig. 8, where we find slightly different exponential constants for the two data sets. Consequently, one would expect $A$ vs $N$ extrapolations to be less reliable than $A$ vs $t$ extrapolations. This is observed in Table $\mathrm{V}$, where linear $N$-type extrapolations [Eq. (2)] give fits that have larger $\chi_{\nu}^{2}$ values than exponential foil thickness extrapolations. Nevertheless, even with this oscillatory behavior, by using $1 / A$ or $A$ vs $N$ fits we obtain extrapolated $A_{0}$ values close to the $A_{\text {true }}$ value with deviations similar to the exponential thickness extrapolations. At high energy, $N$ extrapolations perform as accurately as thickness extrapolations. Extrapolations with $N$ are also useful in that they can be used to determine if the foil is saturated or not, i.e., if the scattered electron count rate (or $A$ ) has reached its asymptotic thickness limit. (In this case, it is fruitless to use a thicker foil to get more signal.) They can also be used to

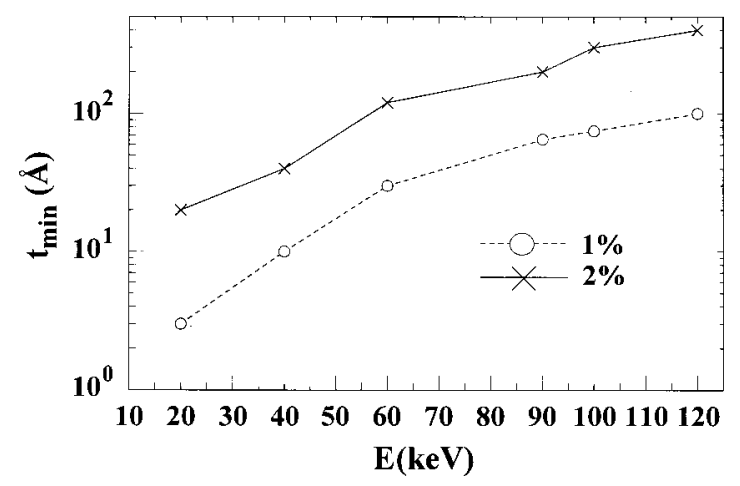

FIG. 12. Estimated minimum $t$ values required, when Eq. (1) is used, to extrapolate to within $(-1 \%$ and $(\times) 2 \%$ accuracy of $A_{\text {true }}$.

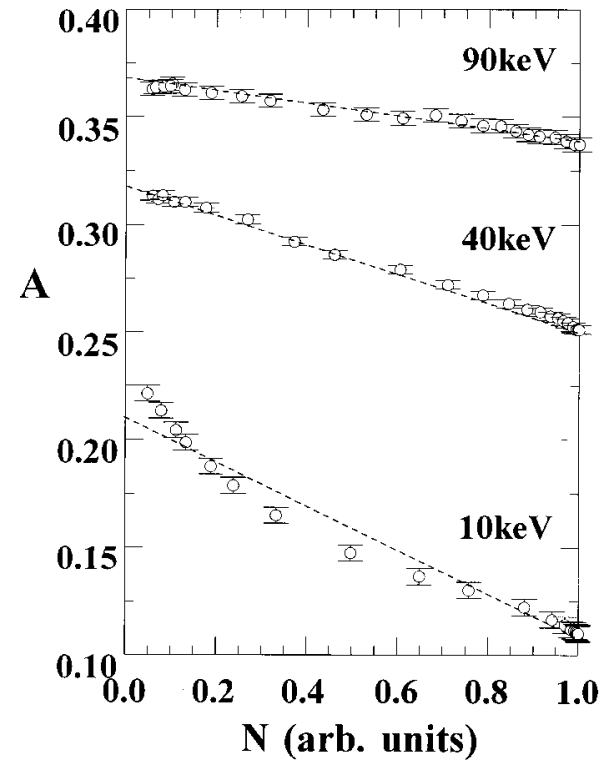

FIG. 13. Plots of $A$ vs $N$ at various $E$ values, for $\Delta \theta=20^{\circ}$. The dotted lines are least-squares fits to Eq. (2). The extrapolated values of $A_{0}$ are given in Table $\mathrm{V}$ together with the corresponding $\chi_{\nu}^{2}$ values.

cross-check the results of asymmetry fits, or used if accurate knowledge of relative foil thicknesses is missing.

(e) Comparison of accuracy of extrapolations with experiment. We compare our $A$ and $1 / A$ vs $N$ and $t$ extrapolations at $E=100 \mathrm{keV}$ with the experimental data taken by Gay et al. [4] in Fig. 14. We have normalized their $A$ and $N$ values to our values at $34 \AA$. We find that linear fits of $1 / A$ and $A$ vs $t$ and $N$ to their data and our data result in mean values of $A_{0}$ equal to $0.374 \pm 0.002(A, 1 / A$ vs $t)$ and $0.373 \pm 0.003(A, 1 / A$ vs $N$; also see Table $\mathrm{V}$ for comparison). These show agreement with the $A_{\text {true }}$ value of 0.374 with less than $1 \%$ uncertainty.

In Fig. 15, we compare 1/Г obtained with Eq. (1) fitted to our BH $\varepsilon=0$ results, $1 / \Gamma^{\prime}[$ Eq. (21)] obtained by fitting $N$ vs $t$, and $\lambda_{e}$ and $\lambda_{i}$ for electrons, derived from the data of Misell

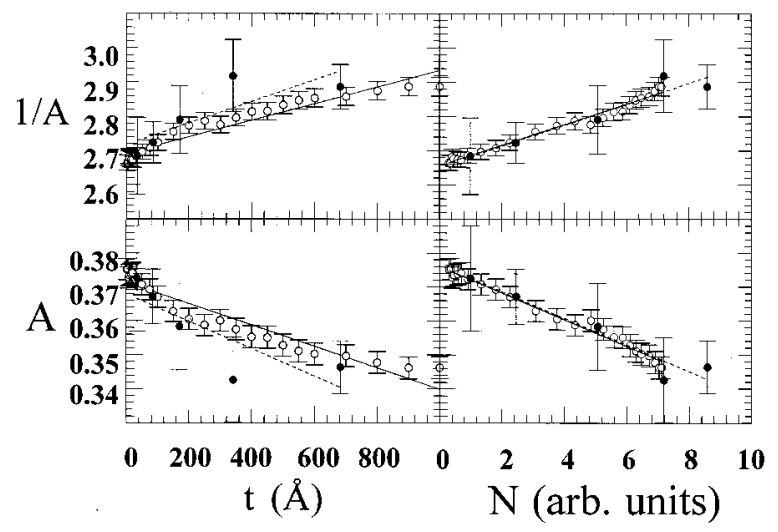

FIG. 14. $A, 1 / A$ vs $t, N$ at $\varepsilon \approx 0$ and $E=100 \mathrm{keV}$. Legend: $\bigcirc$, present work with linear fit (solid line); $\bullet$, experiment of Gay et al. [4] $(\varepsilon=4 \mathrm{eV})$; the dashed line is a linear fit to the experimental values. The experimental data are normalized to our model results at $t=34 \AA$. 


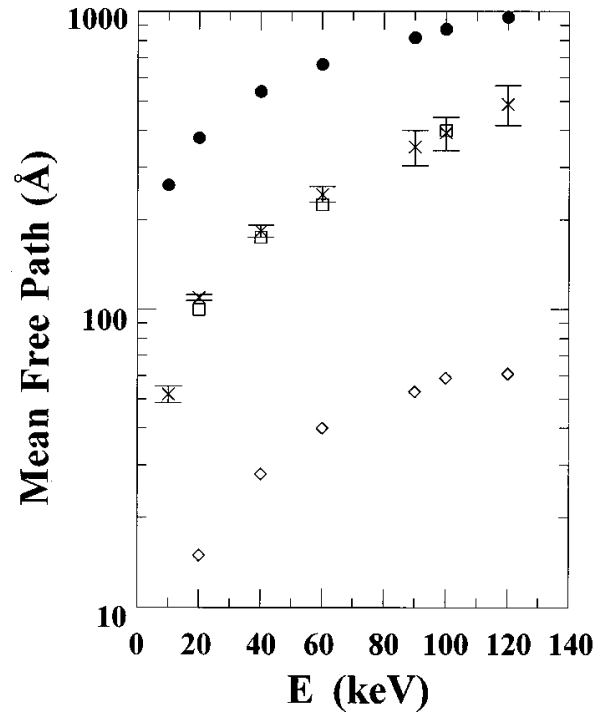

FIG. 15. Comparison of electron scattering mean free paths for elastic and inelastic scattering from gold as a function of $E$. These are taken from Table II or from fits using Eqs. (1) and (19). Legend: $\diamond, \lambda_{e}$ from Ref. [19]; $\bigcirc, \lambda_{i}$ from Ref. [19] using $\sigma_{i} / \sigma_{e}$ ratios of Ref. [24]; average of $\Gamma, \Gamma^{\prime}$ parameters: $\square$, Ref. [4]; $\times$, present work with error bars. See text.

[24] and Ross and Fink [19]. This comparison is made to test an earlier suggestion by Gay et al. [4] that both $1 / \Gamma$ and $1 / \Gamma^{\prime}$ should correspond to $\lambda_{i}$. We observe that our values of $1 / \Gamma$ and $1 / \Gamma^{\prime}$ are in close agreement with each other within error bars, and with those of Gay et al. [4]. Hence in Fig. 15 we compare the average of our $\Gamma$ and $\Gamma^{\prime}$ values with those of Gay et al. Clearly, our average and Gay et al.'s data are very different from both $\lambda_{e}$ and $\lambda_{i}$. In our model we use the $\lambda_{i}$ 's derived from a combination of Misell's and Ross and Fink's data, from which we observe excellent agreement with experimental asymmetry results. We are led to conclude that elastic cross sections, inelastic cross sections, and mean free paths used in our model must be correct, and that $1 / \Gamma$ and $1 / \Gamma^{\prime}$ do not correspond directly to $\lambda_{e}$ or $\lambda_{i}$, in disagreement with Gay et al.'s supposition. We also find that the values of $1 / \Gamma$ and $1 / \Gamma^{\prime}$ lie in between $\lambda_{e}$ and $\lambda_{i}$, showing that the reduction in $A$ and the increase of $N$ with $t$ depend on both elastic and inelastic scattering processes. The former scatters the electrons into/out of the detector, while the latter reduces the electron energy below the detection threshold and causes a loss of elastically scattered electrons.

\section{Dependence of $A$ on $\varepsilon$}

We now consider maximum (cumulative) energy loss $\varepsilon$ extrapolations by including inelastic energy loss. In such cases the extrapolations are done for a fixed $t$ and variable $\varepsilon$. In this model the polarization of the inelastically scattered electron is transformed in the same way as for elastic scattering (Sec. IV A). One should expect a reduction of $A$ as multiple/plural inelastic electrons are allowed to pass the retardation grids, i.e., as $\varepsilon$ is increased. In our calculations $E$ values of 10,20 , and $40 \mathrm{keV}$ were considered, with energy loss bins in the range of $0-1 \mathrm{keV}$ and in increments of $10 \mathrm{eV}$.

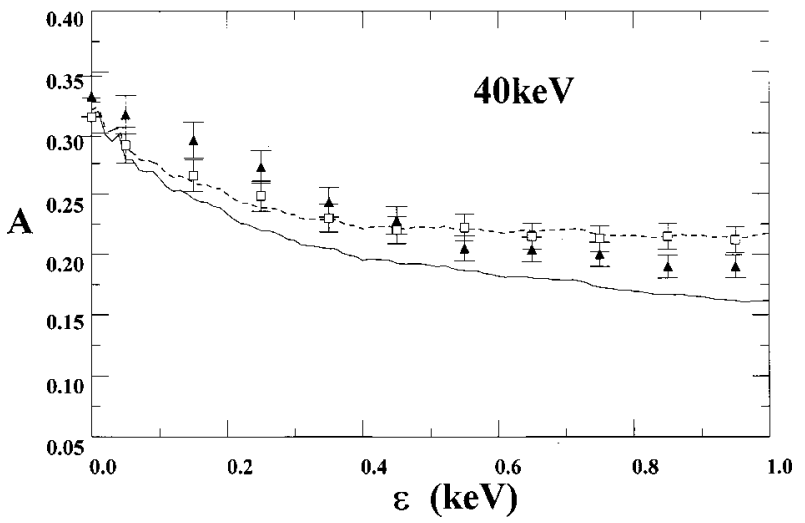

FIG. 16. $A$ vs $\varepsilon$ at $E=40 \mathrm{keV}$. Legend: Experiment, $\square$, Ref. [9] and $\boldsymbol{\Delta}$, Ref. [10]; the solid line is the present model with $100 \%$ electron optical transmission; the dashed line is the present model including an estimate of experimental electron optical transmission (see text).

Only the $40 \mathrm{keV}$ results are reported here. Energy losses greater than $1 \mathrm{keV}$ were placed in the black hole.

In Fig. 16 we compare our $A$ vs $\varepsilon$ results for $E=40 \mathrm{keV}$ and $t=1000 \AA$ with the measurements of Gray et al. [9] and Dunning et al. [10]. Their data were obtained with mini-Mott polarimeters. The exact functional form of $A$ vs $\varepsilon$ depends on experimental specifics. In order to get good agreement with the experimental data, we had to use our $N(\varepsilon)$ curve modified by an approximate estimate of electron optical effects present in their apparatus. We have also used a detection efficiency that rises linearly with $\varepsilon$ as discussed in Refs. [4], [8], and [29]. These results, which agree qualitatively with experiment, give us confidence in the general validity of our energy loss model and inelastic cross-section calculations (Sec. IV C 2). Unfortunately, the widely varying electron optical details of different apparatus make quantitative comparisons between our model and various experimental data sets difficult.

\section{CONCLUSIONS AND SUGGESTIONS FOR FUTURE WORK}

The present work has shown that it is possible to model in a detailed, quantitative way the process of Mott scattering from solid foil targets. The depolarizing effects of multiple and plural scattering have been comprehensively included. From this model, we find the following.

(i) To reliably reach $A_{\text {true }}$ values one needs to observe elastically scattered electrons $(\varepsilon=0)$ and extrapolate to zero foil thickness with extremely thin foils. This means that accurate Mott electron polarimeters require retardation optics placed in front of their detectors (as schematically shown in Fig. 1). This confirms the same conclusion reached by Gay et al. [4]. For $A$ vs $t$ extrapolations with $\varepsilon=0$, exponential forms such as Eqs. (1) and (19) should give accurate extrapolations with errors in principle less than $1 \%$, provided that $E \geqslant 40 \mathrm{keV}$ and the minimum foil thickness is $\leqslant 30 \AA$.

(ii) From a practical viewpoint, several thin foils, all with $t<100 \AA$, are necessary to enable reliable $t$ extrapolations at all energies considered in this paper. For $A$ vs $t$ extrapola- 
tions a recommended, practical selection would be 10, 30, $100,150,250,500$, and $1000 \AA$. Based on our model, these foils would adequately provide $A$ vs $t$ extrapolations with precision in the sub-1\% area for $E \geqslant 40 \mathrm{keV}$.

(iii) The quasiexponential dependence of elastic scattering $A$ values on both $N$ and $t$ is characterized by a decay constant between the inverse of the elastic and inelastic mean free paths.

(iv) In practice, $A_{\text {true }}$ values show significant dependence on the solid angle $\Delta \Omega$ subtended by the detector (Table I). This means that detector solid angles need to be accurately known. Having met this requirement, however, polarimeters have a better detection efficiency when $\Delta \Omega$ is large to optimize their figure of merit, viz., $P^{2} I$ (see [1]).

(v) The functional dependence of $A$ on $\varepsilon$ is complicated by apparatus-specific electron optical considerations. It is thus prudent to use foil thickness extrapolations with rejection of inelastically scattered electrons to determine $A_{\text {true }}$.

We now suggest some possible directions for future experimental and theoretical work relevant to this effort.

(i) Measurements of high-energy differential elastic and either integral or differential inelastic cross sections. These data would be useful for checking the values used in this model.

(ii) Investigations of the validity of other equations for $A$ vs $t$ extrapolations. This would require very precise data, but would result in more accurate extrapolation procedures.

(iii) Measurement of DDCS's using differential energy analyzers as opposed to retarding-field analyzers. This would help the model in determining a correct algorithm for generating these DDCS's, and would improve our modeling of energy loss extrapolations.

(iv) Experimental measurement of $A$ vs $t$ in Mott polarim- eters with large $\left(\Delta \theta>5^{\circ}\right) \Delta \Omega$ values. This could be made, e.g., by using variable apertures placed in front of the detectors (which could be, e.g., multichannel plates). These measurements could be directly compared to our present model with excellent statistics, and would be useful in terms of optimization of the figure of merit of the electron spin polarimeter [1].

(v) Investigation of $A$ vs $N$ behavior with large $\Delta \theta$. This would be very useful in checking the details of our model regarding the marked oscillations at low $E$ values (Fig. 13). However, this requires a large number of closely spaced foil thicknesses or a graded-thickness foil that could be displaced perpendicular to the electron beam.

(vi) The extension of all the above to thorium foils. This is very useful because, unlike gold, the thorium inelastic/ elastic ratios are not available and consequently these must be theoretically estimated. Experimental asymmetry values would also be extremely useful for comparison with our model.

In the future, this work could be accelerated by using faster computers to extend our calculations to higher $E$ and smaller $\Delta \theta$ values.

\section{ACKNOWLEDGMENTS}

This work was supported by the National Science Foundation under Grants No. NSF-RUI-PHY-9205423, No. NSFRUI-PHY-9511549, and No. NSF-PHY-9706530. C.H. and N.M. were supported by the NSF under a Young Scholars Program grant awarded to Dr. E. Streitberger. E.Y. was supported by the Southern California Academy of Sciences. The authors thank Dr. M. Fink and Dr. J. Furst for helpful discussions.
[1] T. J. Gay and F. B. Dunning, Rev. Sci. Instrum. 63, 1635 (1992).

[2] A. Gellrich and J. Kessler, Phys. Rev. A 43, 204 (1991).

[3] S. Mayer, T. Fisher, W. Blaschke, and J. Kessler, Rev. Sci. Instrum. 64, 952 (1993).

[4] T. J. Gay, M. A. Khakoo, J. E. Brand, J. E. Furst, W. V. Meyer, W. M. K. P. Wijayaratna, and F. B. Dunning, Rev. Sci. Instrum. 63, 114 (1992).

[5] G. D. Fletcher, T. J. Gay, and M. S. Lubell, Phys. Rev. A 43, 911 (1986).

[6] K. Jost, in Proceedings of the International Symposium on Correlation and Polarization in Electronic and Atomic Collisions, edited by P. A. Neill, K. H. Becker, and M. H. Kelley, Natl. Inst. Stand. Technol. Spec. Publ. No. 789 (U.S. GPO, Washington, DC, 1990), p. 76.

[7] L. A. Hodge, T. J. Moravec, F. B. Dunning, and G. K. Walters, Rev. Sci. Instrum. 50, 5 (1979).

[8] D. M. Campbell, C. Hermann, G. Lampel, and R. Owen, J. Phys. E 18, 664 (1985).

[9] L. E. Gray, M. W. Hart, F. B. Dunning, and G. K. Walters, Rev. Sci. Instrum. 55, 88 (1984).

[10] F. B. Dunning, L. G. Gray, J. M. Ratliff, F.-C. Tang, X. Zhang, and G. K. Walters, Rev. Sci. Instrum. 58, 1706 (1987).

[11] J. Kessler, Polarized Electrons, 2nd ed. (Springer, Berlin, 1985).

[12] H. Wegener, Z. Phys. 151, 252 (1958).

[13] H. Wegener, Bull. Am. Phys. Soc. 5 (4), 238 (1960).

[14] J. S. Greenberg, D. P. Malone, R. L. Gluckstern, and V. W. Hughes, Phys. Rev. 120, 1393 (1960).

[15] L. Braicovich and B. de Michelis, Nuovo Cimento B 58, 269 (1968).

[16] V. Hnizdo, Nucl. Instrum. Methods 109, 503 (1970).

[17] S. Qiao and A. Kakizaki, Rev. Sci. Instrum. 68, 4017 (1997).

[18] G. Holzwarth and H. J. Meister, Nucl. Phys. 59, 45 (1964); Tables of asymmetry, cross section and related functions for Mott scattering of electrons by screened $\mathrm{Au}$ and $\mathrm{Hg}$ nuclei, University of Munich report 1964 (unpublished).

[19] A. W. Ross and M. Fink, Phys. Rev. A 38, 6055 (1988).

[20] W. H. Press, S. Teukolsky, W. T. Vetterling, and B. P. Flannery, Numerical Recipes in C, 2nd ed. (Cambridge University Press, Cambridge, 1992).

[21] W. H. Press and S. Teukolsky, Comput. Phys. 8, 522 (1992).

[22] American Chemical Society Handbook, 50th ed., edited by R.C. Weast (The Chemical Rubber Publishing Company, 
Cleveland, OH, 1969), Sec. B-24.

[23] E. N. Lassettre, J. Chem. Phys. 43, 4479 (1965).

[24] O. C. Wells, Scanning Electron Microscopy (McGraw-Hill, New York, 1974), Chap. 3; D. L. Misell, Adv. Electron. Electron Phys. 32, 63 (1973).

[25] V. E. Cosslett and R. N. Thomas, Br. J. Appl. Phys. 15, 893 (1964); 15, 1283 (1964).
[26] A. Corney, Atomic and Laser Spectroscopy (Clarendon, Oxford, 1979), Chap. 9.

[27] K. Palik, Handbook of Optical Constants (Academic, New York, 1985), p. 81.

[28] M. Uhrig, A. Beck, J. Goeke, F. Eschen, M. Sohn, G. F. Hanne, K. Jost, and J. Kessler, Rev. Sci. Instrum. 60, 872 (1992).

[29] F. Bordoni, Nucl. Instrum. Methods 97, 405 (1971). 